\title{
A New Strategy For Multi-OBJeCtive Dynamic AND Kinematic Optimization of Robotic MANIPULATORS WITH APPLICATION IN HAPTIC INTERFACES
}

BY: BEHROOZ ALAE

B.Sc. IN MECHANICAL ENGINEERING, TEHRAN UNIVERSITY, TEHRAN, IRAN, 1992

A THESIS SUBMITTED IN PARTIAL FULFILLMENT OF THE REQUIREMENTS OF MASc DEGREE IN MECHANICAL ENGINEERING FACULTY OF ENGINEERING, ARCHITECTURE AND APPLIED SCIENCE RYERSON UNIVERSITY TORONTO, ONTARIO, CANADA

c 2006 
UMI Number: EC53475

\section{INFORMATION TO USERS}

The quality of this reproduction is dependent upon the quality of the copy submitted. Broken or indistinct print, colored or poor quality illustrations and photographs, print bleed-through, substandard margins, and improper alignment can adversely affect reproduction.

In the unlikely event that the author did not send a complete manuscript and there are missing pages, these will be noted. Also, if unauthorized copyright material had to be removed, a note will indicate the deletion.

\section{$\mathrm{UMI}$}

\section{UMI Microform EC53475}

Copyright 2009 by ProQuest LLC

All rights reserved. This microform edition is protected against unauthorized copying under Title 17, United States Code.

ProQuest LLC

789 East Eisenhower Parkway

P.O. Box 1346

Ann Arbor, MI 48106-1346 


\section{Author's Declaration Page}

I hereby declare that I am the sole author of this thesis.

I authorize Ryerson University to lend this thesis or dissertation to other institutions or individuals for the purpose of scholarly research.

Signature: $\quad y$

I further authorize Ryerson University to reproduce this thesis or dissertation by photocopying or by other means, in total or in part, at the request of other institutions or individuals for the purpose of scholarly research.

Signature: 


\begin{abstract}
A New Strategy For Multi-Objective Dýnamic and Kinematic Optimization of a Robotic Manipulator with Application in Haptic Interfaces

Behrooz Alae, Master of Applied Science in

Mechanical Engineering, Ryerson University, May 2006
\end{abstract}

There is an increasing demand for higher performance in modern robotic applications. To meet the need for more accuracy and fast dynamic response, considering inertial effects is necessary.

This thesis proposes a new global multi-objective optimization strategy to tune the geometric and dynamic capabilities of a manipulator. Then, as a case study, the kinematics and dynamic behavior of a five-bar-linkage haptic interface is analyzed and a new design procedure is obtained using a new global and constrained multi-objective technique. The minimax culling algorithm was used to design parameters for optimal kinematics and dynamic dexterity measure. 


\section{Acknowledgement}

I would like to express my gratitude to my supervisor Professor Farrokh Janabi-Sharifi from whom I have tried to learn intuitive and analytical thinking. I will always be his student.

I would like to express my heartfelt thanks to my mother and father, Fatemeh and Ayatollah, for their endless love and to my brother and sister, Behzad and Shahla for their great support. 


\section{Table of Contents}

Author Declaration $\ldots \ldots \ldots \ldots \ldots \ldots \ldots \ldots$

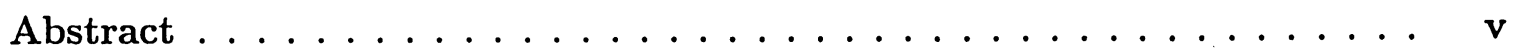

Acknowledgement $\ldots \ldots \ldots \ldots \ldots \ldots \ldots \ldots \ldots$

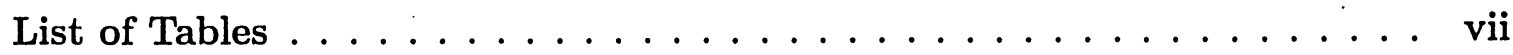

List of Figures $\ldots \ldots \ldots \ldots \ldots \ldots \ldots \ldots \ldots \ldots \ldots \ldots$ vii

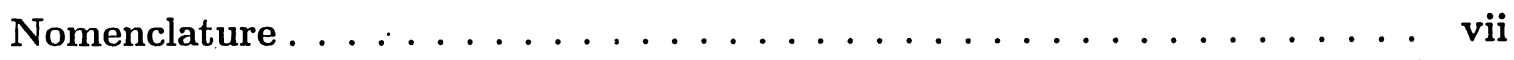

1 INTRODUCTION $\ldots \ldots \ldots \ldots \ldots \ldots \ldots \ldots$

1.1 Previous Work $\ldots \ldots \ldots \ldots \ldots \ldots \ldots$

1.2 Motivation ..................... 4

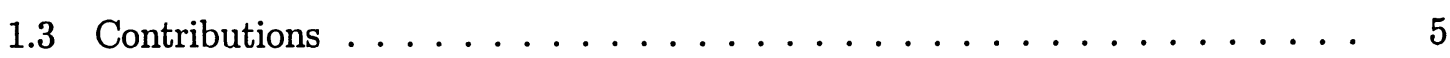

1.4 Organization of The Thesis $\ldots \ldots \ldots \ldots 6$

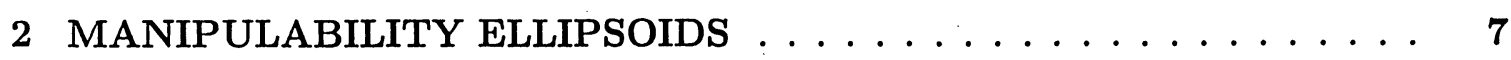

2.1 Kinematic Manipulability Ellipsoids . . . . . . . . . . . 7

2.2 Dynamic Manipulability Ellipsoids _ . . . . . . . . . . . . . 11

3 CULLING OPTIMIZATION ALGORITHM $\ldots \ldots \ldots \ldots$

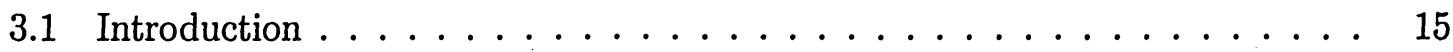

3.2 Minimax Optimization Problem . . . . . . . . . . . . 16

3.3 The Minimax Culling Algorithm . . . . . . . . . . . . . . . 17 
3.4 An Example of a Two Degree of Freedom Minimax Optimization Problem

3.5 Analysis of Culling Algorithm . . . . . . . . . . . . . . . 24

4 KINEMATIC DESIGN OF FIVE-BAR PLANAR HAPTIC DEVICE 26

4.1 General Guideline For Design and Multi-objective Optimization of a Linkage For a Haptic Interface : A Case Study . . . . . . . . . . . . . 26

4.2 Kinematic Design and Optimization of Planar Five-Bar Linkage . . . . . . 28

4.3 Kinematic Optimization Conclusions . . . . . . . . . . . . 31

5 A NEW STRATEGY IN MULTI-OBJECTIVE CONSTRAINT DYNAMIC OPTIMIZATION OF MANIPULATORS .............. 35

5.1 Dynamic Parameter Analysis of a Planar Five-bar Mechanical haptic device " 35

5.2 Global Multi-Objective Minimax Optimization of a Planar Five-bar Haptic

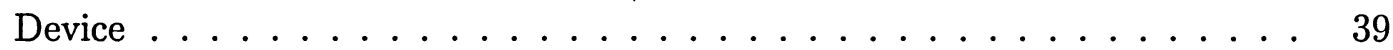

5.3 Constraint Multi-objective Minimax Optimization of a Desktop Planar Fivebar Haptic Device . . . . . . . . . . . . . . . . 42

5.4 Design Constraints For a Desktop 5-bar Haptic Device . . . . . . . . . . 43

5.5 Analysis of The Inertia Parameter On The Dynamic Dexterity Measure (Dimensional Analysis) . . . . . . . . . . . . . . . . 44

5.6 Case Study with New Method . . . . . . . . . . . . . . 46

5.7 Case Study with Stocco's Multi-objective Optimization Method . . . . . . 50

5.8 Comparison Of Proposed Method With Stocco's Method . . . . . . . . . 52

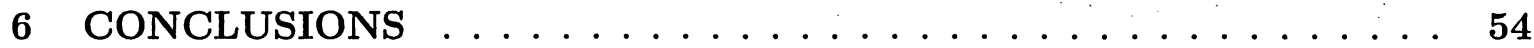

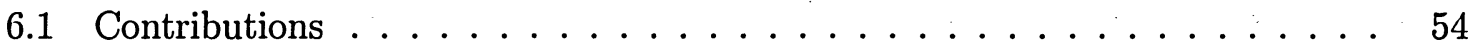

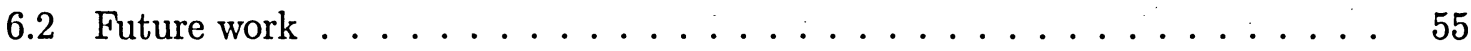

A Permissions .......................663 


\section{List of Tables}

4.1 Reduced parameter space. . . . . . . . . . . . . . . . . 30

5.1 Result of proposed dimensionless optimization analysis of five-bar linkage for

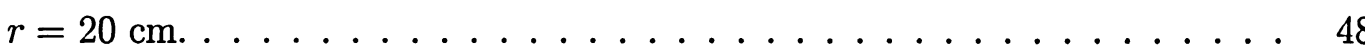

5.2 Comparison of Optimized 5 bar linkage with Stocco and the new method.

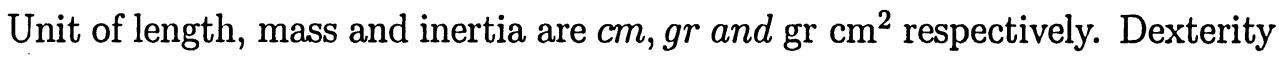
measures are unitless. . . . . . . . . . . . . . . . . 52 


\section{List of Figures}

2.1 Planar two link manipulator and manipulability ellipsoid of end-effector . . 8

2.2 Joint-rate/end-effector ellipses $\ldots \ldots \ldots \ldots \ldots \ldots$

3.1 Saddle point of a two dimensional quadratic function $\ldots \ldots \ldots 17$

3.2 Planar elbow manipulator workspace $\ldots \ldots \ldots \ldots \ldots$

3.3 Kinematic dexterity measure for 2 DOF planar manipulator. . . . . . . . . 22

3.4 Second culling algorithm in branching process. . . . . . . . . . 23

3.5 Second culling algorithm in bounding process. . . . . . . . . . . 24

4.1 A 2DOF planar five-bar linkage $\ldots \ldots \ldots \ldots \ldots$

4.2 Optimal postures of five-bar linkage (With permission from Stocco) [3]. . . . 32

4.3 A schematic wide view of manipulator workspace. . . . . . . . . . 33

4.4 The dexterity and manipulability measure for non-optimal kinematic parame-

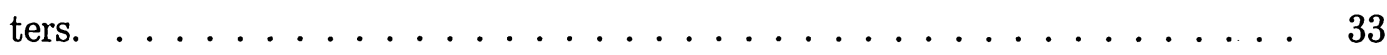

4.5 The dexterity and manipulability measure for an optimal kinematic parame-

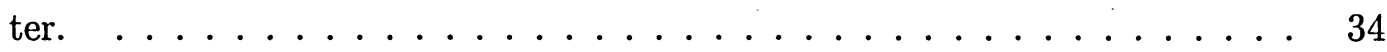

5.1 A 2 DOF planar five-bar linkage $\ldots \ldots \ldots \ldots \ldots \ldots \ldots$

5.2 The proposed dynamic design flowchart of planar five-bar linkage . . . . . 45

5.3 Proposed dimensionless curves for kinematic and dynamic optimization of five-bar mechanism. . . . . . . . . . . . . . . . . 47 
5.4 Dexterity for a non-optimal dynamic parameter; . . . . . . . . . . 48

5.5 Dexterity for an optimal dynamic parameter; . . . . . . . . . . . . . 49

5.6 Multi-objective optimization results from stocco method. . . . . . . . . . 51

5.7 Multi-objective optimization results from Stocco dynamic dexterity definition 53 


\section{Nomenclature}

$\begin{array}{ll}\mathrm{a} & \text { base link length } \\ \mathrm{b}, \mathrm{b} 0, \mathrm{~b} 1 & \text { shoulders link length } \\ \mathrm{c}, \mathrm{c} 0, \mathrm{c} 1 & \text { elbows link length } \\ \mathrm{C} & \text { matrix including centrifugal and coriolis terms } \\ \mathrm{DDM} & \text { Dynamic dexterity measure } \\ \mathrm{G} & \text { matrix including gravity terms } \\ i & \text { looping index } \\ I_{2 o p t} & \text { optimized inertia of elbow link } \\ I_{\text {lopt }} & \text { optimized inertia of shoulder link } \\ I_{2-l o w} & \text { inertia upperbound of elbow link } \\ I_{1-u p} & \text { inertia upperbound of shoulder link } \\ I_{i} & \text { mass moment of inertia of i-th link relative to the end point } \\ \mathrm{J} & \text { Jacobian Matrix } \\ k^{-1} & \text { inverse of condition number } \\ \mathrm{k} & \text { condition number of a matrix } \\ k_{0}, k_{1} & \text { minimum and maximum safety margin } \\ \mathrm{KDM} & \text { Kinematic dexterity measure } \\ \mathrm{L} & \text { ratio of actuator torque to its maximum } \\ \mathrm{M} & \text { inertia matrix } \\ m_{i} & \text { mass of link i } \\ M_{s} & \text { shoulder link inertia matrix } \\ M_{e} & \text { elbow link inertia matrix } \\ m_{\text {min }} & \text { mass per unit length } \\ m_{\text {total }} & \text { total mass of manipulator divided by two } \\ m_{2 o p t} & \text { optimized mass of elbow link } \\ m_{2-l o w} & \text { mass lowerbound for elbow link } \\ m_{1-u p} & \text { mass upperbound of shoulder link } \\ \mathrm{P} & \text { manipulator kinematic parameters } \\ p^{*} & \text { optimum kinematic parameter of the manipulator } \\ P_{i} & \text { set of all the parameter in parameter space } \\ p_{i} & \text { design parameter } \\ & \end{array}$




\section{Nomenclature}

$\hat{p}_{i} \quad$ best known design parameter

q joint Space Configuration of a manipulator

$\dot{\mathbf{q}} \quad$ joint rate of manipulator

$\mathrm{r} \quad$ workspace distance from the origin

$r_{\text {opt }} \quad$ optimized workspace distance from the origin

$s \quad$ performance measure

$\hat{s} \quad$ performance measure of best known design parameter

$\mathrm{V} \quad$ velocity

$W \quad$ set of all positions in workspace

$x \quad$ end-effector position

$\ddot{\mathrm{x}} \quad$ acceleration of end-effector

$\ddot{x}_{\text {vel }} \quad$ acceleration(coriolis part)

$\ddot{\mathbf{x}}_{\text {grav }} \quad$ gravity term of acceleration

$X_{i} \quad$ the distance between the center of mass of link i to its end-point

$\underline{x} \quad$ position with the smallest performance value

$\sigma_{\min } \quad$ minimum singular value

$\sigma_{\max }$ maximum singular value

$\tau_{i}^{\text {limit }}$ upper limit of actuator torque

$\underline{\sigma} \quad$ minimum singular value

$\tilde{\sigma} \quad$ maximum singular value

$\underline{\Sigma}_{i} \quad$ performance measure upperbounding function

$\tilde{\Sigma}_{i} \quad$ performance measure lowerbounding function

$\gamma \quad$ workspace angular angle

$\eta \quad$ workspace deviation

$\sigma_{i} \quad \mathrm{i}$-th singular value of a matrix

$\lambda_{i} \quad$ i-th eigen value of a matrix

$\omega \quad$ kinematic manipulability ellipsoid

$\tau \quad$ actuator torque

$\theta_{i} \quad$ orientation of each link to horizontal axes

$\theta_{1}, \theta_{4}$ the angle between shoulder links and horizontal axes

$\theta_{1}, \theta_{4}$ the angle between elbow 


\section{Chapter 1}

\section{INTRODUCTION}

\subsection{Previous Work}

The world of robotics has entered a stage where the traditional repetitive task of assembling of mechanical elements is just part of robotic applications. Robotics is finding its way in surgerical devices [1], astronomy, teleoperation, training and demonstrations [2] as well as haptic interfaces [3] used for interaction between people and computers. Thus not only workspace size and resolution and payload requirements [4] but also inertial parameter adjustments for mass, inertia and acceleration are important specifications that should be adjusted to do high precision tasks as mentioned above.

Given that the relationship between robot end-effector and actuators varies with position and direction, minimizing this variation and consequently maximizing mechanical isotropy [21] throughout the workspace is highly desirable in modern robotic applications. Studying directional and scale isotropic behavior is really essential for a optimum actuator and controller design [2]. Increasing the isotropy strengthens the worst-case performance of the manipulator and allows smaller actuators to be used, leading to a smaller inertia for whole manipulator [2]. In other words, the robot should perform the task homogenously in different directions. Considerable research has been done to design an isotropic robot in a special 
configuration [5] to move along a trajectory [5] or to explore a workspace [6] [7].

$\mathrm{Ma}$ and Angeles [5] described the concept of dynamic isotropic configurations and tried to optimize geometrical and dynamical parameters for a serial planar three DOF manipulator to find the isotropic configurations of the manipulator. They introduced the dynamic conditioning index (DCI) to quantitatively measure the difference between a given position and the dynamic isotropic configuration [5]. They also applied the concept in the problem of robot trajectory planning and finally they developed a basic theorem about the relation between the kinematic and dynamic pure isotropy for a parallel manipulator in specific isotropic configurations [8]. More research on isotropy and its effect on controller design are presented by Salcudean et al. [9] and a special analytical example of a five-bar haptic device control design was presented as a case-study by Nenchev [10].

To extend the mechanical capability of a manipulator, a suitable measure should be introduced to provide useful information for both the design of multi-purpose robot task as well as planning their efficient motion. Considering the manipulator kinematic properties such as Jacobian, Yoshikawa [11] suggested a measure based on volume of the velocity manipulability ellipsoids. This measure was defined as the set of end-effector velocities which can be performed by joint velocities belonging to a unit sphere. Moreover, according to the duality principle, the force manipulability ellipsoid is defined as the ability of exerting end-effector forces along each task-space direction for a given set of joint torques. Although both ellipsoids principle axes coincide but the lengthes of the axes are inversely proportional. Chiu viewed a manipular as a "mechanical transformer" and introduced the term of task compatibility index as a basis for computing postures that optimize the robot's performance at a special task application [12].

In many applications the manipulator dynamics can not be neglected, which means quantifying dynamic and acceleration capabilities of a robot manipulator is really critical in robot 
design. Yoshikawa [13] proposed another measure, known as the dynamic manipulability measure which incorporates the manipulator mass matrix in addition to Jacobian to find the best dynamic manipulability posture for a planar two-link manipulator. The dynamic manipulability ellipsoids can be imagined as a measure to describe end-effector acceleration with the the joint torques adopted from a unit sphere.

Manipulability ellipsoids are effective tools for performing task space analysis of robotic manipulators. The shape of these ellipsoids demonstrates the ability of the robot to apply velocity, acceleration or force in different directions and various configuration. These measure determine the maximum available performance of a manipulator in a given posture and end-effectors direction of motion. This may be helpful in robotic design where the best manipulator structure and size is needed to execute a given task.

Chiacchio et al. [14] demonstrated that gravity shifts the dynamic manipulability ellipsoid center. Rosenstien [15] investigated the role of velocity, Coriolis and Centrifugal forces on dynamic manipulability . A new definition of dynamic dexterity ellipsoid was proposed by Chiacchio [16] which led to more correct results in evaluating manipulator capabilities in terms of task-space accelerations. Yoshikawa [17] also proposed a method to investigate the compliant motion manipulability for a given compliant motion trajectory.

The haptic interface design is effected by different geometrical, kinematical, dynamical and engineering factors. Since the device is a human-machine interface, it should meet the minimum ergonomic requirement. Device size, inertia, frequency response criteria [18] and workspace size all are the main design factors for the optimum kinematic and dynamic design of a industrial haptic device [7]. The actual size of the mechanism should be around desktop size with minimum possible mass. Also translational motions should occur in the largest portable area within the task-space [7]. Clearly, an optimum kinematical and dynamical design, a multi-dimensional multi-objective optimization method would be required. 
Karadis et al [19] designed a dynamically counterbalanced five-bar linkage for microprobing applications. Hayward et al [7] proposed kinematic optimization of a five-bar mechanism to get a general rough design of a planar 2DOF haptic robotic interface. Stocco introduced a novel approach based on the culling algorithm [6] with a new dynamic dexterity measure to optimize five-bar haptic device both kinematically and dynamically [3]. Design and kinematic optimization of a 5-DOF twin-Pantograph haptic pen [3], twin-Pantograph hybrid manipulator [3], and Stewart platform [6] was presented in reference [2], [22]. A new design matrix normalization technique is presented to cope with the problem of nonhomogeneous physical units for manipulators having both translational and rotational parameters [23],[24].

\subsection{Motivation}

Although a significant research in multi-objective kinematic and dynamic optimization of haptic interfaces was done by Stocco [3], but still the algorithm needs to be modified to challenge. more practical applications. Only rough approximation of inertia matrix was used in [3]. Since the method is dynamically a one dimensional multi-objective optimization technique, parameter space is very small. The method in Stocco [3] ignores multi-dimensional optimization along all possible dynamic parameter space. For example, inertia and mass of each link must be treated independent of each other and of geometry of mechanism (kinematics). Therefore, there is a need to perform dynamic analysis and establish a multidimensional multi-objective method to address the nature of this multi-objective design problem. Also it is necessary to have a good understanding of nature of dynamic equations of 5 bar mechanism for optimization purpose. The optimization technique presented in this thesis is a four dimensional multi-objective optimization problem. The parameter space is big enough, the inertia matrix used for 5 bar linkage is exact, and the results are more 
realistic.

\subsection{Contributions}

In this thesis, a new multi-dimensional multi-objective optimization method is introduced to design a kinematically and dynamically conditioned haptic device. The culling algorithm [3] is used as a global optimization method in place of other existing optimization methods such as Genetic Algorithm [26] which cannot guarantee the global optimum [25] [27] [28] . Also the inertia matrix used for optimization purpose is completely valid with no approximation involved, which could be one of the major contributions of this research. A new vision toward dynamic parameter analysis of five-bar linkage is presented and to avoid the trivial answer involved, with the global optimization a new constrained multi-objective optimization method is proposed based on the design guideline and procedures. During the optimization process, due to the nature of Culling Algorithm, the end-effector may hit positions with bad conditions i.e, self-motion [29],[30] and singularity points [31],[32]. Therefore, having a good knowledge of these concepts presented in respective references would be really crucial in computer programming to avoid divergence in culling algorithm.

In short, the main contributions by this research include:

- Using the exact inertia matrix for 5-bar mechanism in optimization process.

- Presenting a new multi-objective optimization.

- A new dimensionless analysis for dynamic parameters of 5-bar linkage.

- Presenting a new optimal design procedure and guideline. 


\subsection{Organization Of The Thesis}

In chapter 2 , the manipulability and dexterity measure and its importance in robotic design is reviewed. In chapter 3, the culling algorithm as an global optimization algorithm is introduced. chapter 4 discuses culling algorithm application for basic kinematic optimization of a 5 bar linkage mechanism and the design guidelines is derived for haptic devices. Dynamic analysis of a five-bar linkage is presented in chapter 5 and then a new strategy for global multi-objective optimization is used to design a 5 bar haptic interface. The general flowchart of the design process is introduced. The contributions and future research are outlined in chapter 6 . 


\section{Chapter 2}

\section{MANIPULABILITY ELLIPSOIDS}

Design of robotic manipulators requires optimization of their manipulation ability. For this purpose, manipulability and dexterity measures have been developed. In this chapter, measures and analysis associated with the manipulability will be reviewed and the concept of manipulability ellipsoid will be presented as an index characterizing manipulation ability of a robotic manipulator. The manipulability ellipsoid can be defined and used in kinematic and dynamic contexts to graphically demonstrate the manipulation ability of a robotic manipulator in different kinematic and dynamic configurations. The introduced measures and ellipsoids will be utilized in the next chapters for optimizing the five-bar structure.

\subsection{Kinematic Manipulability Ellipsoids}

Consider the planar manipulator in Fig. (2.1) . The Jacobian matrix is a matrix that relates end-effector velocities to the joint rates denoted by $\mathbf{v}$ and $\dot{\mathbf{q}}$, respectively [21]. This relation is defined as follows :

$$
\dot{\mathbf{q}}=\mathbf{J}(\mathbf{q}) \mathbf{v} \text {. }
$$

This matrix generally is a function of the manipulator configuration $q$ and can be assumed as a transmission ratio between the actuator and end-effector. 


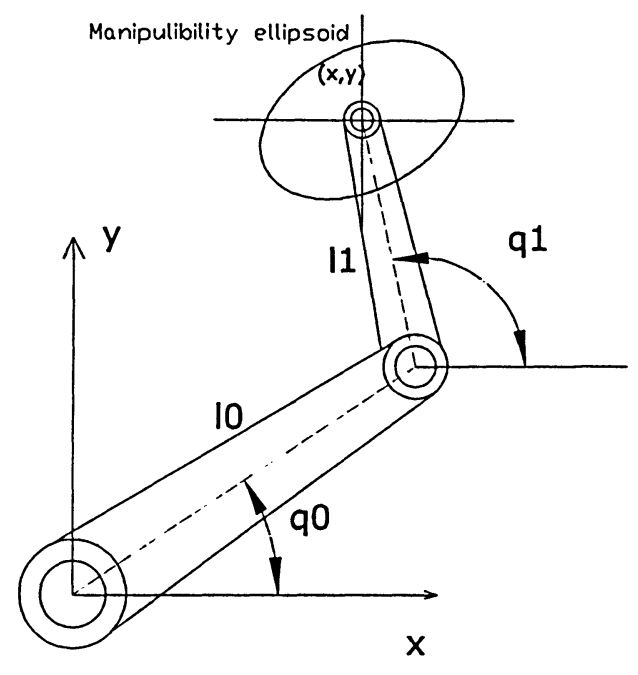

Figure 2.1: Planar two link manipulator and manipulability ellipsoid of end-effector

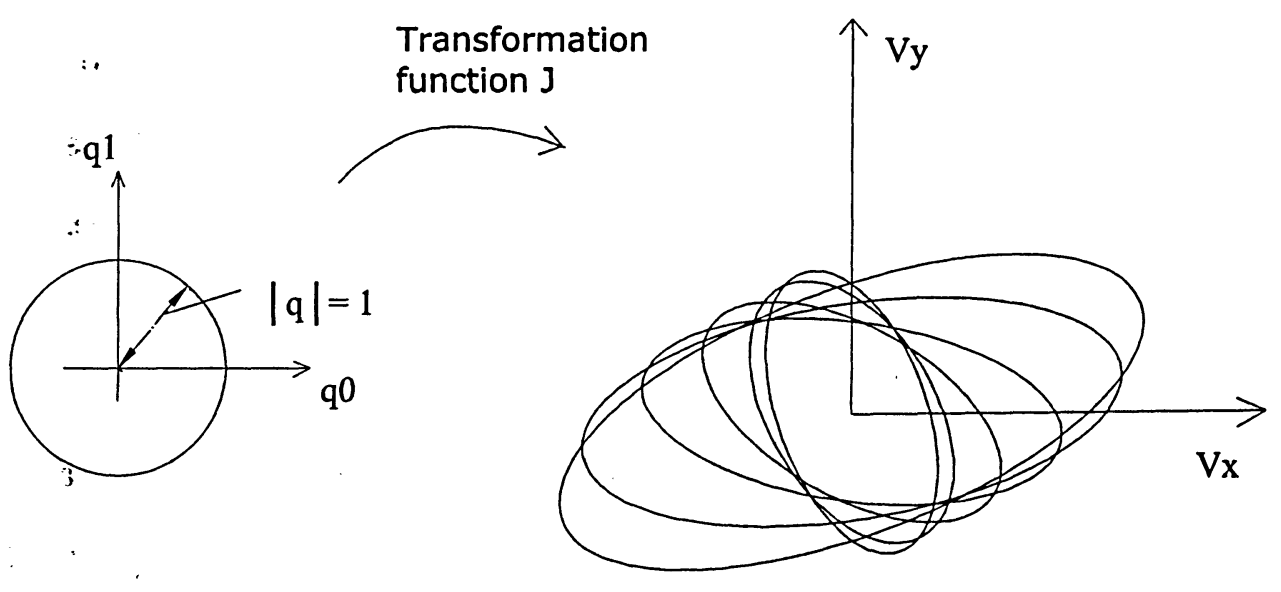

Figure 2.2: Joint-rate/end-effector ellipses 
Consider the set of joint velocities of constant unit norm as follows

$$
\dot{\mathbf{q}}^{T} \dot{\mathrm{q}}=1
$$

The above equation shows all velocity vectors as points on the surface of a unit sphere in the joint velocity space. It is desired to describe the operational space velocities in end-effector that can be generated by the given set of joint velocities. Substitution of eq. (2.1) in eq. (2.2) yields

$$
\mathbf{v}^{T} \mathbf{J}^{T}(\mathbf{q}) \mathbf{J}(\mathbf{q}) \mathbf{v}=1
$$

this ellipsoid describes all the points in the end-effector velocity space and is called kinematic manipulability ellipsoid [21].

As shown in Fig. (2.2), all the point on the surface of the sphere are mapped onto the surface of the ellipsoid in the end-effector velocity space . The kinematic manipulability ellipsoid is a measure that shows direction of dexterous manipulability.

The end-effector can move with large velocity along the direction of the major axis of the ellipsoid, while the smaller end-effector velocities are obtained along the direction of the minor axes. The closer the ellipsoid is to a unit-sphere, the more capable the end-effector is in moving in different directions of the workspace. It is known from basic mathematics that the general geometry of the ellipsoid is highly dependent on the core of quadratic form introduced in eq. (2.3). Also the sharpness and the orientation of ellipsoid depends on the core matrix which is generally a function of configuration. Therefore, the sensitivity of the motion in different directions is dependent on manipulator posture, while shape of manipulability ellipsoid is dependent on the characteristic of its core matrix $\mathbf{J}^{T} \mathbf{J}$. The direction of the principle axes of the ellipsoid are determined by the eigenvectors of the matrix $\mathbf{J}^{T} \mathbf{J}$, while the dimension is completely dependant on singular value of $J$ defined as

$$
\sigma_{i}=\sqrt{\lambda_{i}\left(\mathbf{J}^{T} \mathbf{J}\right)} \quad i=1, \ldots ., n
$$


where $\lambda_{i}\left(\mathbf{J J}^{T}\right)$ denotes the eigenvalues of $\mathbf{J}^{T} \mathbf{J}$. A well-known measure to represent the manipulation ability is the volume of the ellipsoid which is proportional to the following quantity

$$
\omega(\mathbf{q})=\sqrt{\operatorname{det}\left(\mathbf{J}^{T}(\mathbf{q}) \mathbf{J}(\mathbf{q})\right)}
$$

This measure is called Kinematic Manipulity Measure and in the case of non-redundant manipulator it can be reduced to

$$
\omega(\mathbf{q})=\operatorname{det}(\mathbf{J}(\mathbf{q}))
$$

It is concluded from the above definition that the manipulability measure is always nonnegative and is equal to zero in singular points. Therefore, in a way, this measure shows how much the current configuration is far from the singularity configuration.

It is clear that in positions near the singularities, when the manipulator is outstretched, the ellipsoid is very thin along vertical direction. On the other hand, when the manipulator is retracted, the ellipsoid is very thin along the vertical axes.

As it is shown, the eigenvalues approaches zero near the singular configuration. The best advantage of the manipulability measure index is that it can be computed easily through the determinant of $\mathbf{J}^{T} \mathbf{J}$ matrix. On the other hand, since the values computed for manipulability measures cannot demonstrate how close the mechanism is to singularity, this could be imagined as one of the its main disadvantages. For example, in case when different units and scales are used for the links' length, our computation will get different performance results which differ from each other, almost around four order of magnitude.

Therefore, in general, when it is not easy to find a simple and meaningful index for manipulability measure to meet the the above mentioned problems, one can consider the ratio between the minimum and maximum singular values of the Jacobian $\sigma_{\min } / \sigma_{\max }$ as an effective isotropy performance indicator. This index is called dynamic dexterity measure 
and is equivalent to inverse of condition number and gives not only a measure of closeness to singularity configuration but also provides an index to measure the ellipsoid eccentricity directly. On the other hand, the complex relation between this measure and configuration of the manipulator can be considered as one of its major disadvantages. Also, most of the time, it is impossible to find an explicit relationship based on the coordination of the manipulator.

\subsection{Dynamic Manipulability Ellipsoids}

Dynamic manipulability and dexterity is discussed exclusively in [15] and will be completely reviewed in this section for its importance in our research. The equation of motion for an open-loop manipulator with $\mathrm{n}$ rigid links can be expressed as

$$
\tau=\mathbf{M}(\mathbf{q}) \ddot{\mathbf{q}}+\mathbf{C}(\mathbf{q}, \dot{\mathbf{q}})+\mathbf{G}(\mathbf{q})
$$

In above equation effects of disturbances, e.g., friction is ignored in dynamic analysis and $\tau$ is an $n \times 1$ vector of joint actuator torques and $\mathbf{q}, \dot{\mathbf{q}}$ and $\ddot{\mathbf{q}}$ are $n \times 1$ vectors of generalized joint positions, velocities and accelerations respectively. In eq. (2.7), $\mathbf{M ( q )}$ is the $n \times n$ inertia matrix that demonstrates the configuration dependent inertial properties, $\mathbf{C}(\mathbf{q}, \dot{\mathbf{q}})$ represents Coriolis and Centrifugal forces, and $\mathbf{G}(\mathbf{q})$ accounts for the vector of joint torques due to gravity. Also $\mathrm{x}=\left[\begin{array}{llll}x_{1} & x_{2} & \ldots & x_{m}\end{array}\right]^{T}$ represents the m-dimensional task-space vector which introduces the coordinates of the tip of end-effector. In this thesis, it is assumed that end-effector has just translational acceleration and $m \leq 3$. The equations describing the relations between positions from joint space to task space is nonlinear, and the $m \times n$ Jacobian matrix $\mathbf{J}$ represents the first-order term in a Taylor series of the respective mapping.

By differentiating the definition of Jacobian Matrix the corresponding relationship for acceleration is obtained.

$$
\ddot{\mathbf{x}}=\mathbf{J}(\mathbf{q}) \ddot{\mathbf{q}}+\dot{\mathbf{J}}(\mathbf{q}, \dot{\mathbf{q}}) \dot{\mathbf{q}}
$$


Since the mass matrix is positive definite and invertible, the eq. (2.7) can be solved for $\ddot{\mathrm{q}}$ as follows:

$$
\ddot{\mathbf{q}}=\mathrm{M}^{-1}(\tau-\mathrm{C}(\mathbf{q}, \dot{\mathbf{q}})-\mathrm{G}(\mathbf{q})) \text {. }
$$

Substituting eq. (2.9) in eq. (2.8) and dropping the explicit dependencies on $\mathbf{q}$ and $\dot{\mathbf{q}}$ yields:

$$
\ddot{\mathbf{x}}=\mathrm{JM}^{-1}(\tau-\mathrm{C}-\mathrm{G})+\dot{\mathrm{J}} \dot{\mathrm{q}}
$$

It is notable that the result is in terms of actuator torques rather than joint accelerations and can be simplified to:

$$
\ddot{\mathbf{x}}=\mathrm{JM}^{-1} \tau+\ddot{\mathbf{x}}_{v e l}+\ddot{\mathbf{x}}_{\text {grav }},
$$

where

$$
\ddot{\mathbf{x}}_{v e l}=-\mathrm{JM}^{-1} \mathrm{C}+\dot{\mathrm{J}} \dot{\mathrm{q}}
$$

and

$$
\ddot{\mathbf{x}}_{\text {grav }}=-\mathrm{JM}^{-1} \mathrm{G} \text {. }
$$

we assume that torque limits are symmetric as in ref. [16] such that

$$
-\tau_{i}^{l i m i t} \leq \tau_{i} \leq-\tau_{i}^{l i m i t}, i=1, \ldots \ldots ., n
$$

then we can introduce $\tilde{\tau}$ as a normalized actuator torque

$$
\tilde{\tau}=\mathrm{L}^{-1} \tau_{i}
$$

where $\mathrm{L}=\operatorname{diag}\left(\tau_{1}^{\text {limit }}, \ldots . ., \tau_{n}^{\text {limit }}\right)$ and with the help of eq. $(2.14)$, a limit for admissible torques is defined as:

$$
\|\tilde{\tau}\|_{\infty} \leq 1
$$


Changing eq. (2.11) to the new form with normalized actuator torques denoted as $\tilde{\tau}$ yields:

$$
\begin{aligned}
\ddot{\mathbf{x}} & =\mathrm{JM}^{-1} \mathbf{L} \tilde{\tau}+\ddot{\mathbf{x}}_{v e l}+\ddot{\mathbf{x}}_{g r a v} \\
& =\mathrm{JM}^{-1} L \tilde{\tau}+\ddot{\mathbf{x}}_{b i a s}
\end{aligned}
$$

where the bias acceleration defined as $\ddot{\mathbf{x}}_{\text {bias }}$ in following equation.

$$
\ddot{\mathbf{x}}_{b i a s}=\ddot{\mathbf{x}}_{v e l}+\ddot{\mathrm{x}}_{\text {grav }}
$$

To have a better imagination about the $\ddot{x}_{b i a s}$, we can imagine it as the end-effector acceleration while $\tilde{\tau}=0[15]$.

The inequality $\|\tilde{\tau}\|_{\infty} \leq 1$ introduces an n-dimensional hypercube which is mapped by eq. (2.18) to an m-dimensional polytope that delimits the set of feasible end-effector accelerations (the bias term is ignored for simplicity). Alternatively, eq. (2.18) can be imagined as a matrix transformation of the $\mathrm{n}$-dimensional sphere defined by

$$
\tilde{\tau}^{T} \tilde{\tau} \leq 1
$$

to an m-dimensional ellipsoid. This dynamic manipulability ellipsoid is derived by solving eq. (2.18) for $\tilde{\tau}$ and substituting the results into eq. (2.20):

$$
\left(\ddot{\mathrm{x}}-\ddot{\mathrm{x}}_{\text {bias }}\right)^{T}\left(\mathrm{JM}^{-1} \mathrm{~L}\right)^{-T}\left(\mathrm{JM}^{-1} \mathrm{~L}\right)^{-1}\left(\ddot{\mathrm{x}}-\ddot{\mathrm{x}}_{\text {bias }}\right) \leq 1 .
$$

Since the inertia matrix and $\mathrm{L}$ are representing a symmetric matrix, the final inequality for dynamic manipulability ends up to the following equation:

$$
\left(\ddot{\mathrm{X}}-\ddot{\mathrm{X}}_{b i a s}\right)^{T}\left(\mathrm{~J}^{-T} \mathrm{ML}^{-2} \mathrm{MJ}^{-1}\right)\left(\mathrm{X}-\ddot{\mathrm{X}}_{b i a s}\right) \leq 1 .
$$

The shape of the dynamic manipulability ellipsoid can be determined by the matrix $\mathrm{J}^{-\mathrm{T}} \mathrm{ML}^{-2} \mathrm{MJ}^{-1}$ which is determined by previous equation. 
On the other hand, in case when the actuator torque limits are the same, $\mathrm{L}$ matrix reduces to identity matrix which indicates that there is no need for actuator torque limits [15]. The ellipsoid principle axes is specified by the eigenvectors $v_{i}$ of manipulability matrix and the length of the eigenvectors is equal to $1 / \sqrt{w_{i}}$, where $w_{i}$ is the corresponding eigenvalues. On the other hand, the shape of kinematic manipulability ellipsoids can be defined by $\mathbf{J}^{-T} \mathbf{J}^{-1}$, ignoring the manipulator's inertia and actuator torque limits.

The dynamic dexterity measure [15] (DDM) can be introduced as:

$$
\mathrm{DDM}=\frac{\underline{\sigma}\left(\mathrm{J}^{-1} \mathrm{M}\right)}{\tilde{\sigma}\left(\mathrm{J}^{-1} \mathrm{M}\right)}
$$

where matrix $\mathbf{J}$ is Jacobian matrix, $\mathbf{M}$ is inertia matrix of the manipulator and $\underline{\sigma}$ and $\tilde{\sigma}$ represents minimum and maximum eigenvalues, respectively. 


\section{Chapter 3}

\section{CULLING OPTIMIZATION ALGORITHM}

\subsection{Introduction}

In previous section, dexterity measure is introduced as a good quantity to measure how well a robotic manipulator can react in different configurations while satisfying a designated performance function. Maximizing kinematic or dynamic dexterity measure is the main goal for optimization and there is just a few algorithm that can address the optimization problem. For the functions such as condition number or dexterity measure, since the objective function is highly nonlinear, discrete, discontinuous and even unbounded the optimization problem is more complicated. Descent algorithms which usually use differentiation of the objective function go to local optimum points and there is no clear definition for stopping criteria [3]. The performance of global search gets more poor as the search resolution gets smaller.

In the next section the nature of a typical minimax problem is discussed. The culling algorithm as an effective method to deal with optimization problem is discussed in section 3.3. This new algorithm guarantees finding the same results as global search but ends up to the same solution with fewer steps in algorithm. The culling algorithm basically searches through the sub-optimal parameter space, evaluating all its elements and then culls the 
non-optimal elements until it just ends to one element which naturally is the global optimal element. Then in section 3.4, a two degree of freedom minimax problem is discussed to show a more sensible geometrical view to the optimization process.

\subsection{Minimax Optimization Problem}

Basically the minimax optimization problem arises when mathematically we are looking for saddle points of a function. Consider a function with two variable such as

$$
y=A+(x-B)^{2}-(p-C)^{2}
$$

A three dimensional view is illustrated in Fig. (3.1). As it can be checked from basic calculus Point $\mathrm{A}(\mathrm{b}, \mathrm{c})$ is the saddle point of the function and its magnitude is equal to the value a. According to mathematical definition, the saddle point is acting as minimum point in one direction such as $\mathrm{x}$ axis and maximum in other direction such as $\mathrm{p}$ axis. So if we are starting from an initial condition such as point $\mathrm{A}(\mathrm{x}, \mathrm{p})$, with the help of following algorithm the process of searching ends up to saddle point and eventually the culling algorithm will be introduced as follows.

1. Set $P(x, p)$ as initial point on the surface.

2. Repeat the algorithm until points $P$ and $M$ gets to the same point.

3. Move along $x$ direction with a fixed parameter $\mathrm{p}$ to find the minimum point as $N$.

4. Move along $p$ direction with a fixed position $\mathrm{x}$ to find the minimum point as $C$.

5. Go to 2

Ideally when we have a two-variable quadratic function it just take two step to reach to optimal point and for more complex functions the process of optimization will take more 


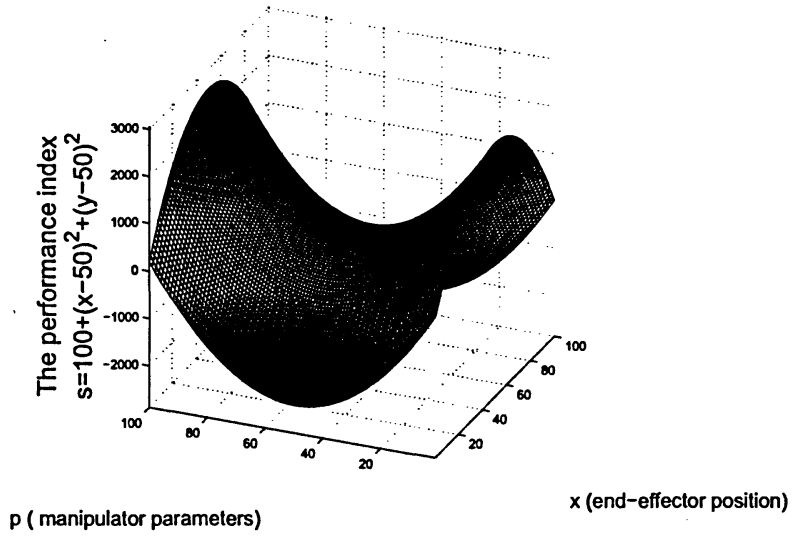

Figure 3.1: Saddle point of a two dimensional quadratic function

steps. This algorithm makes a lot of sense in the problems called "best worst-case scenario". In these type of problems for finding the worst-case scenario we have to minimize a function and after finding the minimum we have to try to maximize that minimum to get to an overall good performance over a defined workspace. More discussion is done in section 3.3 where a optimization problem is explained for a simplified example of a two degree of freedom planar manipulator.

\subsection{The Minimax Culling Algorithm}

In this research, minimax culling optimization algorithm is introduced as as a very useful form of manipulator optimization technique. Culling algorithm is basically a discrete optimization algorithm that belongs to the branch and bound family of optimization algorithms [3]. The algorithm checks all the parameter space and identifies the non-optimal ones and culls them from the parameter domain, resulting a domain with just one element which represents the saddle point or the minimax point of the function.

Hayward [7] used dynamic dexterity measure as the objective function $s(p, x)=\frac{\sigma(\mathbf{D}(p, x))}{\overline{\tilde{\sigma}}(\mathbf{D}(p, x))}$ 


\begin{tabular}{ll} 
& \multicolumn{1}{c}{ List of symbols } \\
$P_{i}$ & looping index \\
$p_{i}$ & set of all the parameter in parameter space \\
$\hat{p}_{i}$ & design parameter \\
$W$ & best known design parameter \\
$x$ & set of all positions in workspace \\
$\underline{x}$ & end-effector position \\
$\underline{\Sigma}_{i}: P_{i} \longrightarrow \Re$ & position with the smallest performance value \\
$\tilde{\Sigma}_{i}: P_{i} \longrightarrow \Re$ & maximum performance measure upper bounding function \\
$s$ & performance measure \\
$k^{-1}$ & inverse of condition number \\
$\hat{s}$ & performance measure of best known design parameter
\end{tabular}

to optimize a planar pantograph haptic interface. This performance measure is not workspace inclusive and is a local function which make it easy for us to apply the new minimax optimization algorithm.

Dynamic dexterity measure index varies from zero to one as the manipulator travels from a singular point to ideally a best possible performance point over a workspace $\mathrm{W}$ and it is desired to find $p^{*}$ which is the best worst-case behavior of the manipulator throughout the whole the workplace.

$$
p^{*}=\arg \max \min s(p, x)
$$

Minimax culling algorithm is specially designed to solve such a minimax problem. The algorithm is introduced in following flowchart from steps 1 to 10 .

\section{$\underline{\text { Minimax Culling Algorithm }}$}

1. set $i=0, \quad s_{0}=\tilde{\Sigma}_{0}=0$

2. $\underline{\Sigma}_{0}(p)=1 \quad ; \forall p \in P_{0}$

3. Choose any $\left(p_{0}=\hat{p}_{0}\right) \in P_{0}$

While $\quad \hat{p}_{i} \neq p_{i}$ 
4. Find $\underline{x}_{i}=\arg _{x \in W} \min s\left(x, p_{i}\right)$

5. if $\quad \hat{s}_{i+1}=s\left(p_{i}, x_{i}\right)>\hat{s}_{i} ; \hat{p}_{i+1}=p_{i}$

otherwise $\quad ; \hat{p}_{i+1}=p_{i} ; \hat{s}_{i+1}=\hat{s}_{i}$

6. Set $\underline{\Sigma}_{i}(p)=\min \left(\underline{\Sigma}_{i}(p), s\left(p, \underline{x}_{i}\right)\right) ; \forall p \in P_{i}$

7. Set $P_{i+1}=\left\{p \in P_{i} \mid \underline{\Sigma}_{i+1}(p)>\hat{s}_{i+1}\right\}$

8. choose $p_{i+1} \in \arg _{p \in P_{i+1}} \max \underline{\Sigma}_{i+1}(p)$

9. $i=i+1$

End

As it is shown in the algorithm the best known performance measure is assumed zero and the algorithm contains a looping index. The algorithm starts with an optimistic bounding functions (step 2) and an initial $p_{0}$ is chosen arbitrarily from $P_{0}$ (step 3). At each point of the workspace the performance measure can be computed from the Inertia matrix for that specific manipulator parameters and the worst performance posture of the manipulator will be obtained.(step 4) Then readily if the minimum performance measure generates a better performance index than the best known parameter $p_{i}$, it will be introduced as the new best known parameter $p_{i+1}$ and a new best known performance measure $s_{i+1}$ will be calculated (step 5). In step (6) performance measure is calculated for each $p$ in $P_{i}$ at fixed $\underline{x}_{i}$ and the upper bound of optimal design will be updated. In step 7 the parameter with the performance worse than new upper bound will be culled from our search domain. As it is shown in the flowchart of algorithm, the parameter with the largest performance measure is transferred as the new optimum candidate $p_{i}$ in step 8 . All the step between 2 to 10 are repeated and in each iteration, culling algorithm will cancel part of parameter domain and 
this process will continue until just one global optimum element remain in the parameter domain which is the global optimum answer.

Since the algorithm is designed in a way, that just elements with singular value ratios worse than other elements will be culled from the domain, the global optimum is guaranteed. Moreover, as it is shown in algorithm flowchart, by keeping a constant search strategy, the culling algorithm is exploring the whole workspace in different configurations and gradually gets close to a sub-optimal space which is the key for convergence of the algorithm to optimum element [3]. Culling algorithm also exhibit a good efficiency due to the fact that in a bounded continuous range of parameter the algorithm remain continuous and holds more or less same quality of performance in that neighborhood. In another word, if the manipulator acts poorly in a configuration, mostly around that neighborhood, the quality of the operation of robot, nevertheless kinematically or dynamically, would be more or less the same. Therefore, if an specific point is culled from the parameter space, more likely the points in its neighborhood would be the best candidate to be culled from parameter space when evaluated at that position.

\subsection{An Example of a Two Degree of Freedom Minimax Optimization Problem}

Consider the planar elbow manipulator of Fig. (3.2). In this example, a two dimensional geometrical concept of the algorithm is introduced to visualize the optimization process and have a general sense about the efficiency and reliability of the minimax culling algorithm. The example is illustrated in a good perspective to show how the algorithm converges. The performance index is $s(x, p)=\frac{\sigma(\mathrm{J}(x, p))}{\tilde{\tilde{\sigma}}(\mathrm{J}(x, p))}$, the one that Hayward [7] used for examining the manipulability of a two degree of freedom planar pantograph kinematic. For better geometrical imagination, it is necessary to reduce the parameter space to one dimension as 
well as the workspace dimension. Therefore, a constraint will be introduced to ensure that the boundaries of usable and reachable workspace are separated well enough. $k_{0}$ and $k_{1}$ are two safety margins to keep the manipulator away from the singularity points in internal and external boundaries shown in Fig. (3.2). Also a minimum safety margin of length $\mathrm{K}$ is introduced as a general safety margin such that $\left[k_{0} k_{1}\right] \geq K$ and $l_{2}$ is defined as [6]

$$
l_{2}=\max \left(\left\|\sqrt{x_{\max }^{2}+y^{2} \|},\right\| y-l_{1} \|\right)+K \text {. }
$$

where $x_{\max }, y, l_{1}, l_{2}$ are shown in Fig. (3.2). The Jacobian matrix of such a system is defined as [33]

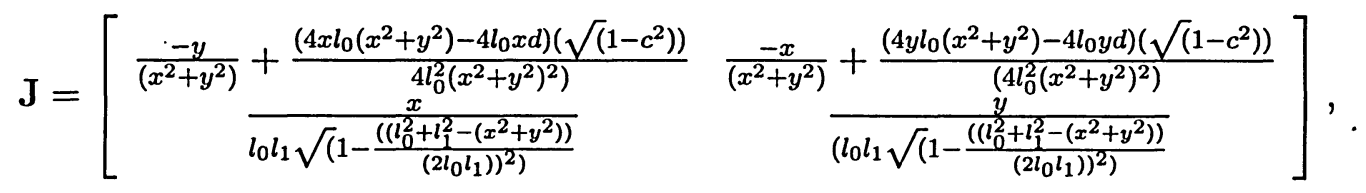

where $l_{0}$ and $l_{1}$ are link lengths. As it can be recognized from above equation that the performance index resulting from this matrix is highly nonlinear, non-differentiable and contains local minima and maxima. Assuming $x_{\max }=5, y=2, K=0.4$ and $2 \leq l_{1} \leq 8$, this index is plotted in Fig. (3.3) against two variables, one working in operational space denoted as $\mathrm{x}$ and the another is $\mathrm{p}$ in parameter space.

The value of $l_{1}=6$ is selected as an initial condition for the best known parameter denoted (step 3). Then the workplace of $l_{1}=6$ is searched to find the configuration that makes the $s(x, p)$ minimum (step 4$)$ which is $s(.4,6)=.28$ Fig. (3.4). As it is clear by this process the condition index is updated by (step 5). Now the parameter space at the configuration with $x=0.4$ will be searched, and the upperbound $\widetilde{\Sigma}_{1}$ will get updated for each parameter (step 6), then parameters that have poor performance (non-optimal space)

- are culled from the original parameter space (step 7). $l_{1}=2.9$ is the maximum point for $s(x, p)$ curve while configuration is fixed at $x=0.4$ (step 8$)$. In the next step the workspace of the $l_{1}=2.9$ is explored (step 4 again) and $s(x, p)$ reaches its maximum at $s(5,2.9)=.16$ 


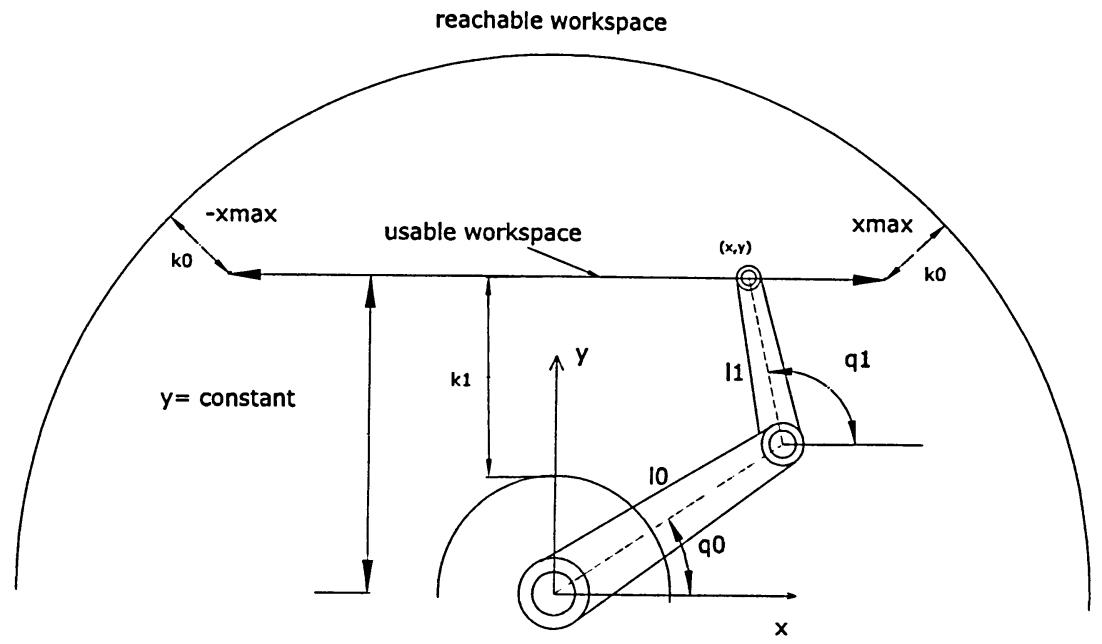

Figure 3.2: Planar elbow manipulator workspace

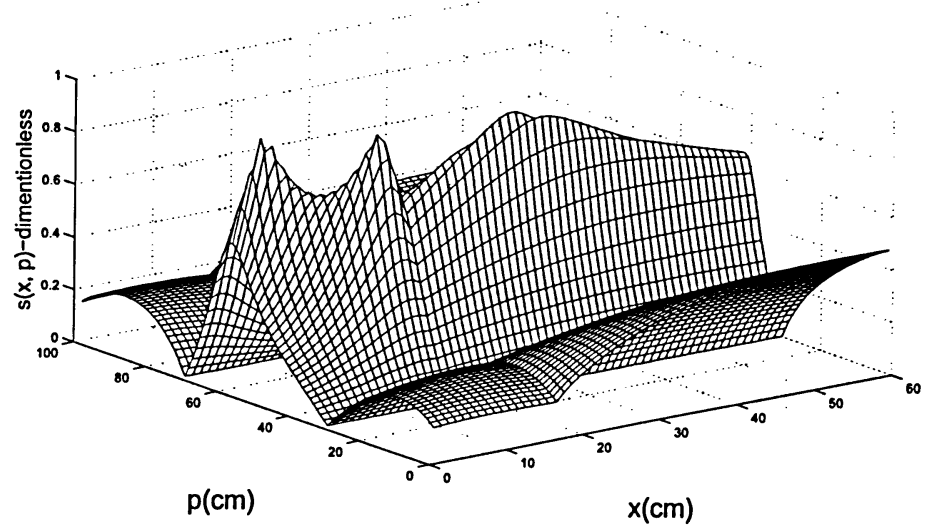

Figure 3.3: Kinematic dexterity measure for 2 DOF planar manipulator. 


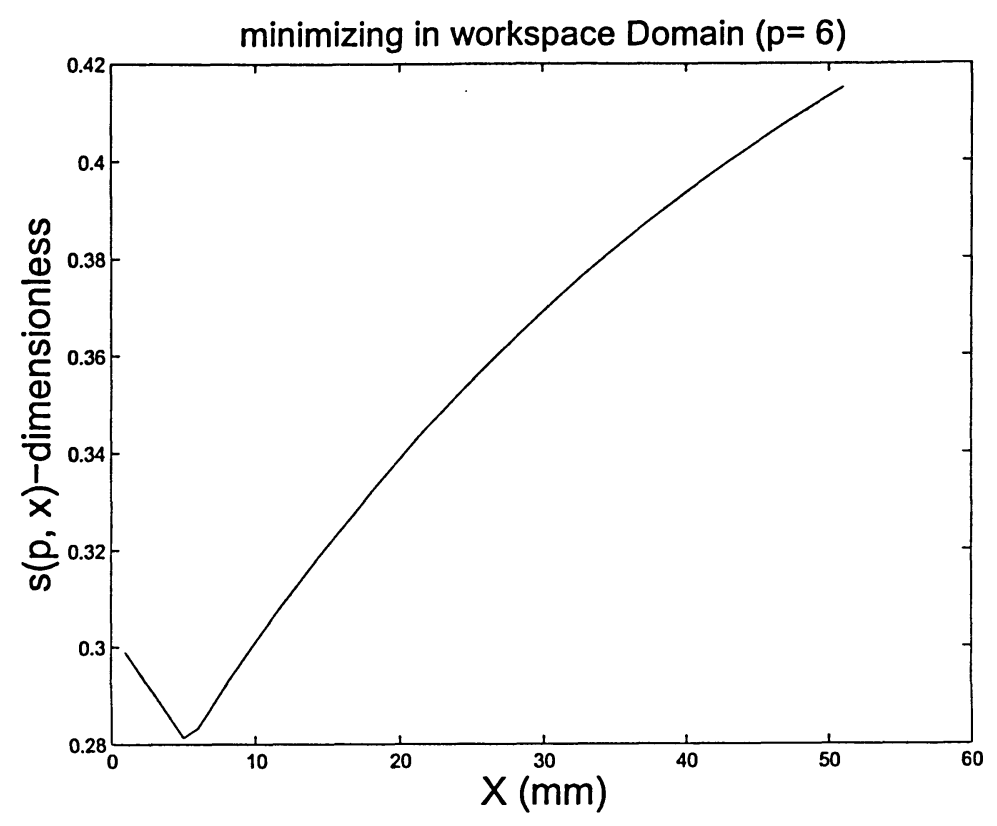

Figure 3.4: Second culling algorithm in branching process.

which is lower than the old lowerbound .28 in Fig. (3.4 ). As there is no improvement in index due to step $5, l_{1}=2.9$ is still the best known parameter (step 5) and again the parameter space at $x=5$ will be searched and the upperbound $\widetilde{\Sigma}_{2}$ Fig. (3.5) is updated from the remaining part of the parameter space (step 6 and eq. (3.5)). Finally after 3 iteration saddle point of the $s(p, x)$ is found at the configuration $s(0,4.5)=.41$.

$$
\underline{\sum}_{2}\left(l_{1}\right)=\min \left(\underline{\sum}_{1}\left(l_{1}\right), s\left(l_{1},-5\right)\right)=\min \left(s\left(l_{1}, 0\right), s\left(l_{1},-5\right)\right)
$$

At the last iteration, due to the fact that all the upper bounds belonging to remaining part of the parameter space are below this value and they are culled from the parameter space and the algorithm will end up to a remaining parameter space with just one element and finally the global optimum $p^{*}$ is obtained. 


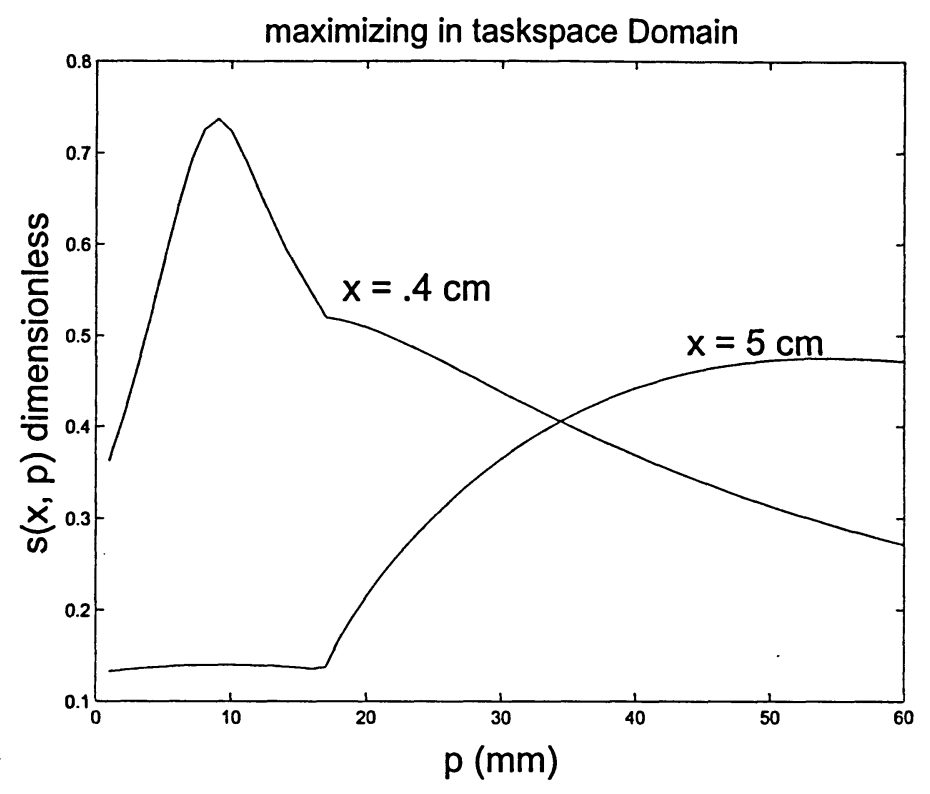

Figure 3.5: Second culling algorithm in bounding process.

\subsection{Analysis of Culling Algorithm}

The culling algorithm is specially designed for solving minimax problems and it is essentially part of family of optimization algorithm named branch and bound. In the process of bounding there is no need to any worst-case estimate of the objective function and the algorithm works through explicit function evaluations [3]. The algorithm evaluates the condition index of a candidate parameter, then the computed value is used to push up the lower bound . Also for each new position, the condition number is computed for different parameter, and the value is used to push down the upper bound. When the value of the lower bound on the optimal performance index exceeds the upper bound on the performance index of any parameter, then the algorithm culls that parameter from the parameter space.

The process of branching is also the other essential part of the culling algorithm where it can go to different direction exploring all the variables by switching between workplace 
variable optimization to parameter space variable optimization and visa versa to end up to an integrated culling strategy. Simply in branching part of the algorithm, there is couple of successive alternation between workspace searches with fixed parameter and parameter space searches with a fixed position while the parameter and position is inherited from the result of previous iteration. It is essential to note that the result of the exhaustive global search will end up to the same global minimum obtained from culling algorithm in a more time consuming process [3]. 


\section{Chapter 4}

\section{KINEMATIC DESIGN OF FIVE-BAR PLANAR HAPTIC DEVICE}

Five-bar haptic device has a lot of applications in designing surgery devices. Karidis [1] introduced a balanced five-bar mechanism for micro probing. The basic design vision on kinematic optimization of a five-bar planar haptic device was presented by Hayward et al. [7]. Stocco [3] introduced a new definition of global isotropy and re-examined the kinematic and dynamic properties of a five-bar mechanism to achieve a dynamically and kinematically optimal design of a manipulator.

\subsection{General Guideline For Design and Multi-objective Optimization of a Linkage For a Haptic Interface : A Case Study}

There are many factors affecting the design of optimized linkage for a haptic device. Factors such as size, workspace, intrusion, inertia, response and structural properties are considered as the most important parameters influencing the design measures. The relationship between these parameters is discussed by Hayward [7]. As a haptic interface is essentially a human 
machine interface, it must have the general features of an ergonomic design. In particular, the linkage for haptic applications should be compact and the workspace has to have a big size compared to the whole task space size. The haptic device should be designed in a way to fit on a table and its size should be about the size of a book [7]. On the other hand, having minimum intrusion in the work area of the user is another qualifying factor for the best design and usually it is difficult to quantify. However, quantitatively a low profile design compatible with the human hand would be the best choice [7]. Thus the size relations are the first general indicators of performance. Inertia could be another concern too. Practically, by considering the latest technology for designing the actuators and selecting the lightest design, an ideal inertia of fraction of a gram throughout a workspace (of the order $.01 \mathrm{~m}^{2}$ ) [7] seems unreachable. Therefore, our trial will be focused on minimizing the inertia of the whole mechanism.

As it is shown in basic dynamics, first natural frequency is really a critical parameter for dynamic design. Therefore, the response of a haptic device, operating below first natural frequency, will be governed by its multi-body dynamics.The frequency of output stimulator should be far enough from the first natural frequency. On the other hand, the frequency response must be wide enough since humans are known to perceive force stimuli well above $300 \mathrm{~Hz}$ [7]. The manipulator should be designed in a environment free of backlash, friction, and other disturbing dynamics [7]. Although feedback and feedforward techniques can be applied to compensate for variation of transfer function, the expense for such a controller and suitable actuators could be high [2] and by making the design matrix such as Jacobian or inertia matrix more isotropic, the better and cheaper design for controller and smaller actuator can be achievable [2]. 


\subsection{Kinematic Design and Optimization of Planar Five- Bar Linkage}

Hayward [7] examined the five-bar linkage based haptic interface by using the inverse of condition number as dexterity measure and introduced the general strategy for optimizing the five-bar mechanism. Using culling algorithm and new definition of global isotropy as kinematic dexterity measure, Stocco [3] established a more detailed research about the planar five-bar mechanism dynamics and kinematics.

The performance of a robot is highly affected by many parameters such as robot geometry and workplace position. As shown in Fig. (4.1), the workspace shape is assumed to be square with length $w$ and position of the robot's workspace are represented by two parameters $(r, \gamma)$ as polar coordinate of center of workspace and $\eta$ represents the angle of the workspace from the horizontal axes (workspace orientation). The main motivation for introducing $\gamma$ and $\eta$ as workspace parameters is to investigate symmetric properties of the device and to obtain for future design optimization. The geometry of the mechanism is defined by three parameter $a, b$ and $c$ representing the length for three links. The length of the ground link is represented by parameter $2 a$; shoulder link by parameter $b$ and elbow link by parameter $c$. To create an environment with more robotic applications and more compatibility to different tasks, non of these parameter is allowed to be zero.

In the formulation $(4.1),\left(\dot{q}_{0}, \dot{q_{1}}\right)$ act as input to the haptic device and $(\dot{x}, \dot{y})$ represent the output of the mechanism in the end-effector. The Jacobian matrix is the matrix that explains the relation between these inputs and outputs. As shown in eq. (4.1) to eq. (4.9) [3], the Jacobian matrix is function of position and link lengths. 


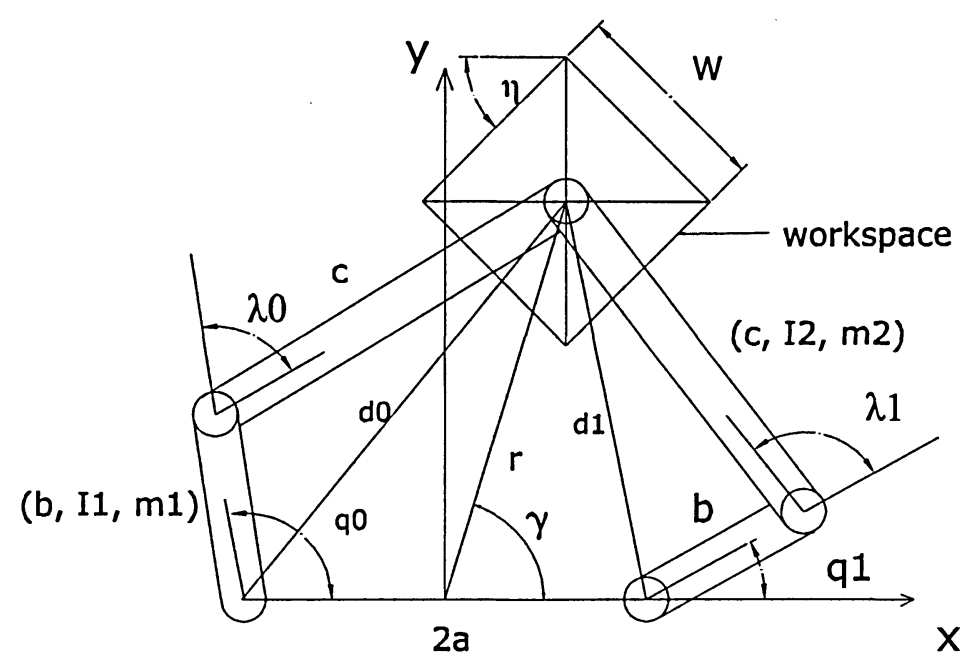

Figure 4.1: A 2DOF planar five-bar linkage

$$
\begin{aligned}
& {\left[\begin{array}{l}
\dot{q}_{0} \\
\dot{q}_{1}
\end{array}\right]=\mathrm{J}(p, x)\left[\begin{array}{c}
\dot{x} \\
\dot{y}
\end{array}\right]} \\
& d_{0}=(x+a)^{2}+y^{2} \\
& d_{0}=(x+a)^{2}+y^{2} \\
& d_{1}=(x-a)^{2}+y^{2} \\
& J_{0}=b_{0}^{2}-c_{0}^{2}-d_{0} \\
& J_{1}=b_{1}^{2}-c_{1}^{2}-d_{1} \\
& J_{2}=d_{0} \sqrt{4 b_{0}^{2} d_{0}-\left(b_{0}^{2}-c_{0}^{2}+d_{0}\right)^{2}} \\
& J_{3}=d_{1} \sqrt{4 b_{0}^{2} d_{0}-\left(b_{0}^{2}-c_{0}^{2}+d_{0}\right)^{2}} \\
& \mathbf{J}=\left[\begin{array}{cc}
-\frac{y}{d_{0}}+\frac{J_{0}(x+a)}{J_{2}} & \frac{x+a}{d_{0}}+\frac{J_{0} y}{J_{2}} \\
-\frac{y}{d_{1}}-\frac{J_{1}(x-a)}{J_{3}} & \frac{x-a}{d_{1}}-\frac{J_{1} y}{J_{3}}
\end{array}\right]
\end{aligned}
$$




\begin{tabular}{|c|c|c|c|c|}
\hline parameter & Min. val & Max. val. & Resolution & optimized results \\
\hline $\mathrm{a}$ & 0 & $15 \mathrm{~cm}$ & $2 \mathrm{~mm}$ & $\mathrm{a}$ \\
\hline$b_{0}, b_{0}$ & $5 \mathrm{~cm}$ & $30 \mathrm{~cm}$ & $2 \mathrm{~mm}$ & $b_{0}=b_{0}=b$ \\
\hline$c_{0}, c_{1}$ & $5 \mathrm{~cm}$ & $30 \mathrm{~cm}$ & $2 \mathrm{~mm}$ & $c_{0}=c_{1}=c$ \\
\hline$\gamma, \eta$ & 0 & $\pi / 2$ & $\pi / 20$ & $\gamma=90 ; \eta=0$ \\
\hline
\end{tabular}

Table 4.1: Reduced parameter space.

The isotropy is dependant on $\frac{\Delta(w)}{r}[3]$ which is the ratio between resolution of movement in the workspace and $r$ (which shows the overall mechanism size). Since isotropy improves when $r \longrightarrow \infty$ or $w \longrightarrow 0$, to avoid trivial answers, the $w$ and $r$ should be fixed in the whole process of optimization. Stocco [3] investigated effect of variation of all the seven independent parameter $\left(a, b_{0}, b_{1}, c_{0}, c_{1}, \gamma, \eta\right)$ and proved that the center of optimized workspace should be located on y axis $\left(\gamma=90^{\circ}\right)$ and the workspace has no horizontal deviation angle $(\eta=0)$. As we mentioned before, all throughout the process two parameters are fixed $(w=10$ and $\Delta(w)=0.1)$. Moreover it was shown that the global optimization has the right and left symmetry of both robot and workspace and different shoulders and elbows have the same length $\left(b_{1}=b_{0} ; c_{1}=c_{0}\right)$. Therefore, for furthermore optimization just only three parameter $\left(a, b=b_{0}=b_{1}, c=c_{0}=c_{1}\right)$ need to be optimized in half of the operational workspace $(x \geq 0)$ [3]. All mentioned stooco results is shown in table (4.2) [6].

In the next step to get to more practical manipulator, Stocco [3] optimized the link length of a symmetric five-bar planar haptic device kinematically and dynamically [3]. Using culling algorithm. Result of kinematical optimization for different locations of the workspace while constraining the elbow angles $\lambda_{0}, \lambda_{1} \in\{0 \ldots . \pi\}$ is illustrated in Fig. (4.2) [3]. Fig. (4.3) shows a typical view of the workspace assuming $r=10 \mathrm{~cm} ; w=10 \mathrm{~cm}$ and Table 4.1 represents the reduced parameter space [3] used during the optimization. Also Fig. (4.4) shows the dexterity and manipulability measure for a non-optimal parameter such as $a=5 \mathrm{~cm} ; b=11 \mathrm{~cm} ; c=17 \mathrm{~cm} ; r=15 \mathrm{~cm}$. Fig. (4.5) also shows the 
dexterity and manipulability measure for manipulator in the optimal point introduced as $a=4 \mathrm{~cm} ; b=9 \mathrm{~cm} ; c=15 \mathrm{~cm} ; r=15 \mathrm{~cm}$.

As it can be examined from Fig. (4.2) the optimized manipulator can be categorized to 4 general postures depending on how far the workspace is from the base.

1. elbow up: $\quad 10<r<25$

2. "M" posture: $-8<r<10$

3. star posture: $-12<r<-8$

4. elbow in: $\quad-25<r<-12$.

\subsection{Kinematic Optimization Conclusions}

As it is clear on Fig. (4.2), Kinematic Dexterity curve is non-smooth and the optimal parameter curves $(a, b$ and $c$ ) are discontinuous in $r$. These discontinuities happens in special locations on the Kinematic Dexterity curve when there is a switch from one posture to other. It can be concluded that although the performance curve is almost level in both star and $\mathrm{M}$ postures, the dexterity measure is not high enough and link lengths are longer than other postures which should be avoided. Elbow-up and elbow-in postures have good dexterity measures comparing to other two postures while elbow-out postures have smaller link lengths. Therefore, it seems that more compact mechanisms can be constructed by elbow-out postures having a relatively high acceptable dexterity measure.

So from perspective of kinematic optimization, " $\mathrm{M}$ " posture is the best and the dexterity measure increases when $r \longrightarrow \infty$. On the other hand, due to ergonomic constraints introduced by hayward [7] the mechanism, should be assembled on a desktop and hold a space around a book which indirectly introduce upper constraint for $r$ and consequently upper 


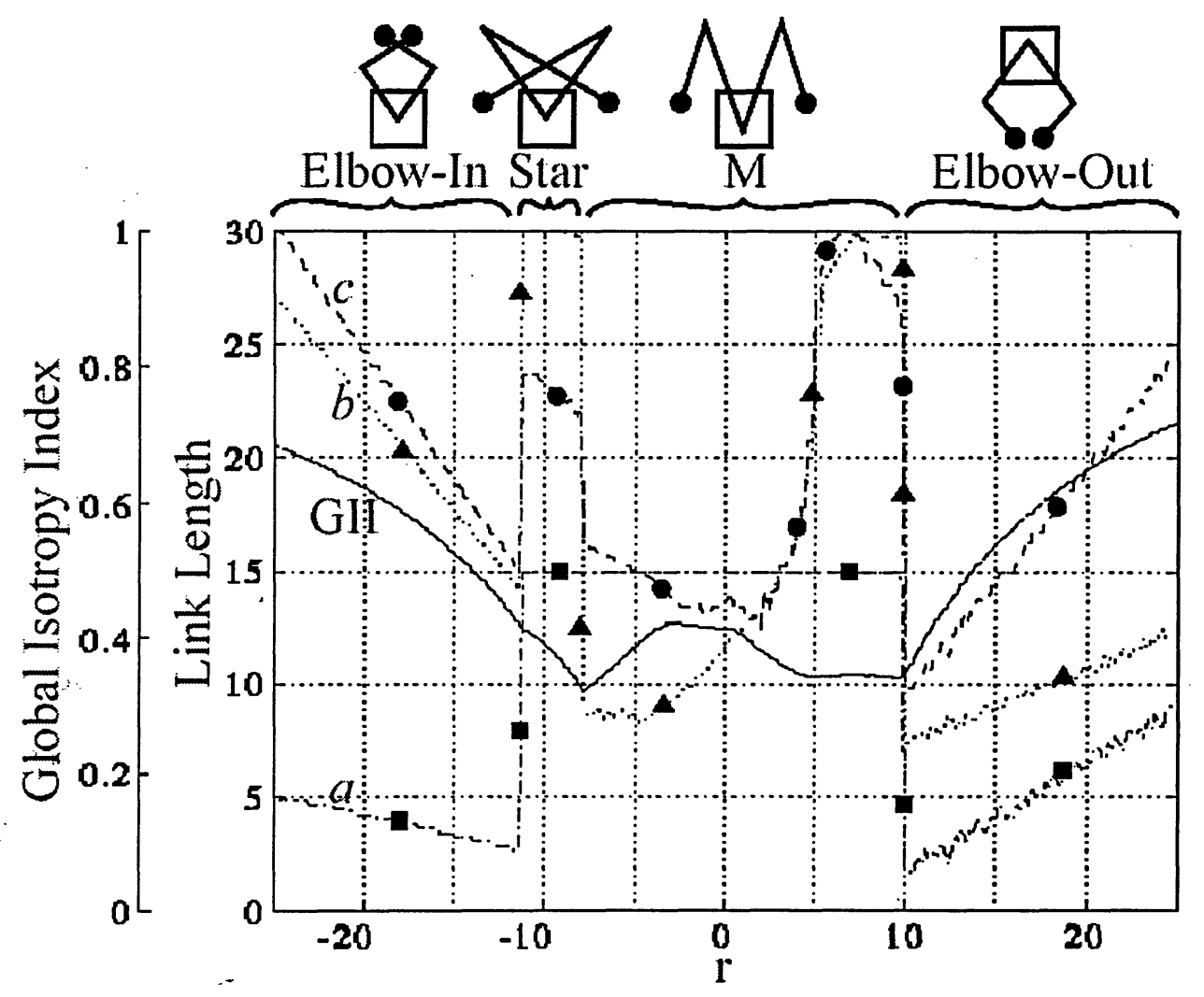

Figure 4.2: Optimal postures of five-bar linkage (With permission from Stocco) [3]. 

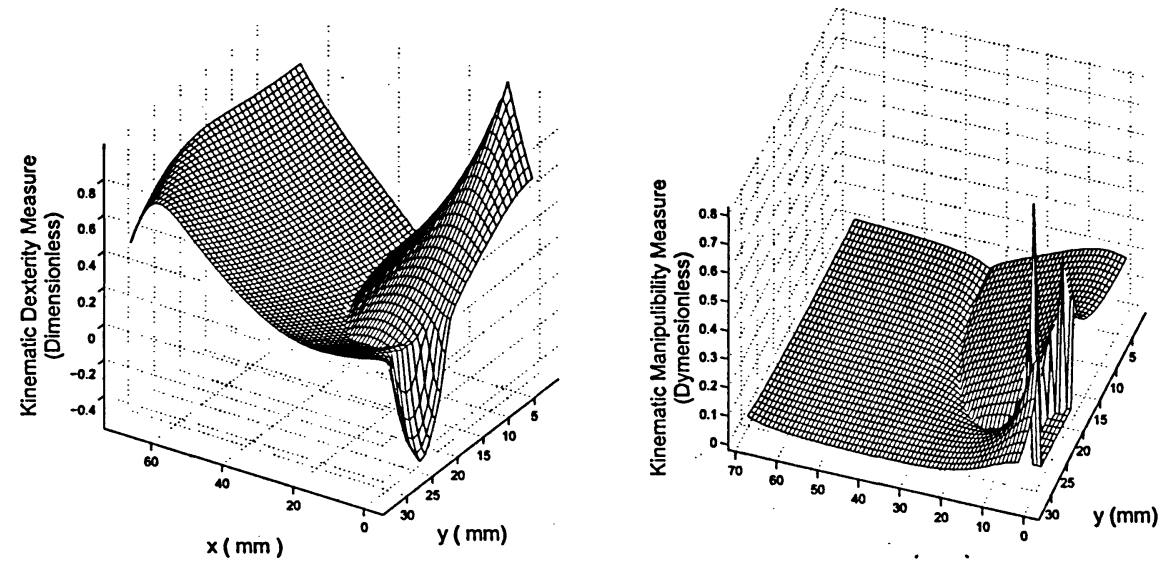

Figure 4.3: A schematic wide view of manipulator workspace.
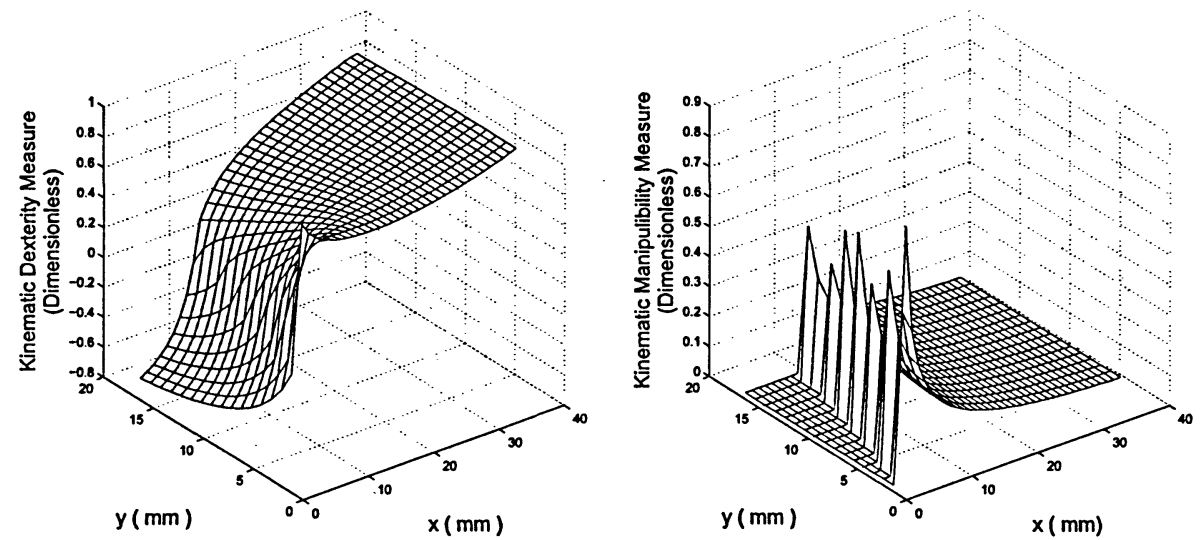

Figure 4.4: The dexterity and manipulability measure for non-optimal kinematic parameters.

limit for maximum dexterity. So it can be concluded that all the elbow-out postures with $r \geq 10$ (when workspace width is 10) are in optimal space depending on $r$ which is the distance between workspace and the base. In other words, the global optimum point for kinematic optimization exists and it can be obtained as $r \longrightarrow \infty$. A more natural method of keeping device sizes reasonable is introduced in new multi-objective optimization method introduced in next section. 


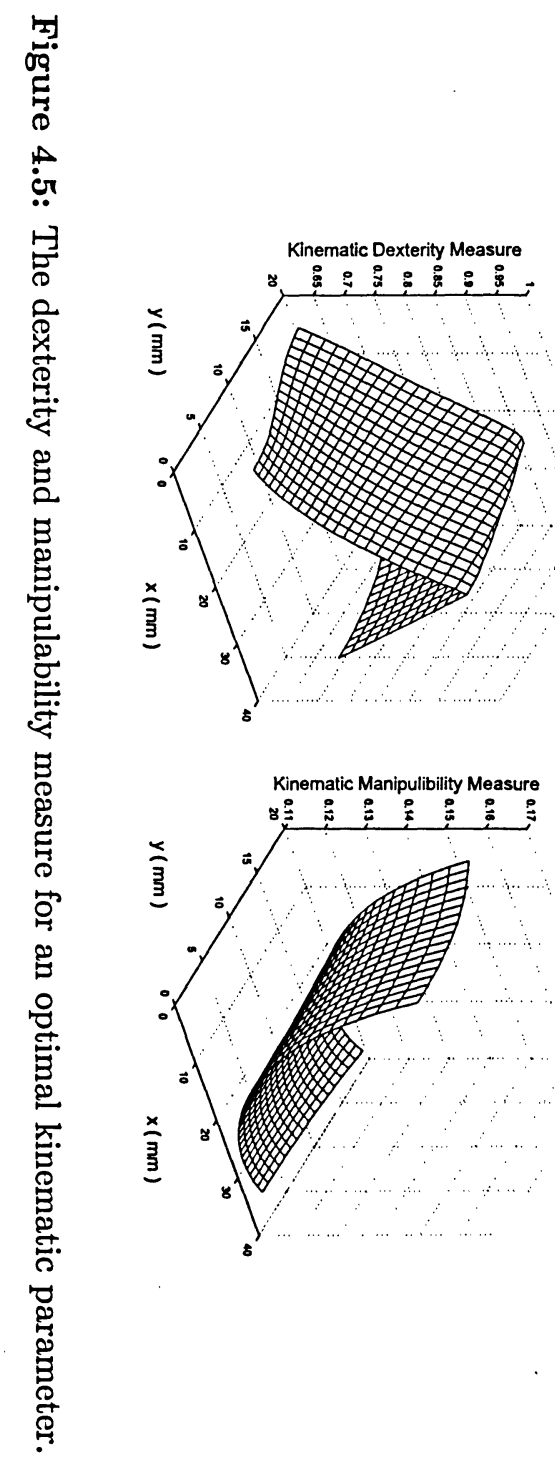




\section{Chapter 5}

\section{A NEW STRATEGY IN MULTI-OBJECTIVE CONSTRAINT DYNAMIC OPTIMIZATION OF MANIPULATORS}

Stocco managed to optimize 5 bar linkage kinematically [3]. To avoid complexity of multi dimensional multi-objective dynamic optimization, he introduced relationship between geometric and inertial parameters and ended up with a one dimensional dynamic optimization problem [3] which is too simplified and is not so realistic. In this chapter, a new method for constraint multi-objective multi-dimensional dynamic and kinematic optimization is presented and the planar five bar linkage is used as a basic planar parallel manipulators for our case study.

\subsection{Dynamic Parameter Analysis of a Planar Five-bar Mechanical haptic device}

Inertia matrix of a planar five-bar haptic device was presented by Kazerooni [34] in (5.1) to (5.9) as follows:

$$
M=\left[\begin{array}{ll}
M_{11} & M_{12} \\
M_{21} & M_{22}
\end{array}\right]
$$


where:

$$
\begin{aligned}
& M_{11}=I_{1}+I_{2} P_{13}^{2}+I_{3} P_{12}^{2}+m_{2}\left[b_{0}^{2}+2 X_{2} b_{0} \cos \left(\theta_{1}-\theta_{2}\right) P_{13}\right] \\
& M_{12}=I_{2} P_{13} P_{43}+I_{3} P_{12} P_{42}+P_{43} \cos \left(\theta_{1}-\theta_{2}\right) X_{2} b_{0} m_{2}+P_{12} \cos \left(\theta_{4}-\theta_{3}\right) X_{3} b_{1} m_{3} \\
& M_{21}=M_{12} \\
& M_{22}=I_{4}+I_{2} P_{43}^{2}+I_{3} P_{42}^{2}+m_{3}\left[b_{1}^{2}+2 X_{3} b_{1} \cos \left(\theta_{4}-\theta_{3}\right) P_{42}\right]
\end{aligned}
$$

$P_{13}, P_{43}, P_{12}, P_{42}$ are given as follows:

$$
\begin{aligned}
& P_{13}=\frac{b_{0} \sin \left(\theta_{1}-\theta_{3}\right)}{c_{0} \sin \left(\theta_{2}-\theta_{3}\right)} \\
& P_{43}=\frac{b_{1} \sin \left(\theta_{4}-\theta_{3}\right)}{c_{0} \sin \left(\theta_{2}-\theta_{3}\right)} \\
& P_{12}=\frac{b_{0} \sin \left(\theta_{1}-\theta_{2}\right)}{c_{1} \sin \left(\theta_{2}-\theta_{3}\right)} \\
& P_{42}=\frac{b_{1} \sin \left(\theta_{4}-\theta_{2}\right)}{c_{1} \sin \left(\theta_{2}-\theta_{3}\right)}
\end{aligned}
$$

Different parameters used in the inertia matrix are as follows:

$M \quad$ the inertia matrix

$I_{i} \quad$ mass moment of inertia of link $i$ relative to the end point

$m_{i} \quad$ mass of link $i$

$X_{i} \quad$ the distance between the center of mass of link $i$ to its end-point.

$b_{1}, b_{0} \quad$ the length of link 1 and 4 or length of the shoulder links

$c_{1}, c_{0} \quad$ the length of link 2 and 3 or length of the elbows

$\theta_{i} \quad$ orientation of each link with respect to horizontal axes

$\theta_{1}, \theta_{4}$ the angles between shoulder links and horizontal axis

$\theta_{1}, \theta_{4}$ the angles between elbow links and horizontal axis

All kinematical and dynamical properties of the mechanism is assumed to be symmetric to the y axis to establish a more simplified model for the inertia matrix. Therefore, the dynamic 
and geometrical properties of the mechanism should satisfy the following equations.

$$
I_{2}=I_{3} ; I_{1}=I_{4} ; m_{2}=m_{3} ; X_{2}=X_{3}=X ; b_{0}=b_{1}=b ; c_{0}=c_{1}=c ;
$$

Applying the above assumptions to the inertia matrix introduced in (5.1), the following simplified inertia matrix is obtained.

$$
\begin{aligned}
& M_{11}=I_{1}+I_{2}\left[P_{13}^{2}+P_{12}^{2}\right]+m_{2}\left[b^{2}+2 X b \cos \left(\theta_{1}-\theta_{2}\right) P_{13}\right] \\
& M_{12}=I_{2}\left[P_{13} P_{43}+P_{12} P_{42}\right]+m_{2}\left[P_{43} \cos \left(\theta_{1}-\theta_{2}\right)+P_{12} \cos \left(\theta_{4}-\theta_{3}\right)\right] X b \\
& M_{21}=M_{12} \\
& M_{22}=I_{1}+I_{2}\left[P_{43}^{2}+P_{42}^{2}\right]+m_{2}\left[b^{2}+2 X b \cos \left(\theta_{4}-\theta_{3}\right) P_{42}\right]
\end{aligned}
$$

where $\theta_{1}, \theta_{2}, \theta_{3}, \theta_{4}$ can be defined as a function of end-effector coordinates $(x, y)$ as follows.

$$
\begin{aligned}
& \theta_{1}=\arctan (y+r, a+x)+\arccos \frac{b^{2}+(y+r)^{2}+(x+a)^{2}-c^{2}}{2 b \sqrt{(y+r)^{2}+(x+a)^{2}}} \\
& \theta_{2}=\arccos \frac{b^{2}+c^{2}-(y+r)^{2}-(x+a)^{2}}{2 b c}-\left(\pi-\theta_{1}\right) \\
& \theta_{3}=\pi-\left(\arccos \frac{b^{2}+c^{2}-(y+r)^{2}-(x-a)^{2}}{2 b c}-\theta_{4}\right) \\
& \theta_{4}=\arctan (y+r, x-a)-\arccos \frac{b^{2}+(y+r)^{2}+(x-a)^{2}-c^{2}}{2 b \sqrt{(y+r)^{2}+(x-a)^{2}}}
\end{aligned}
$$

where arctan is the same as function atan2 in Matlab software. By substituting eqs (5.15) to (5.18) into eqs (5.11) to (5.14), we can transform the inertia matrix from coordinates $\left(\theta_{1}, \theta_{4}\right)$ to coordinates $(x, y)$ as follows:

$$
M=\left[\begin{array}{cc}
I_{1}+I_{2} f_{1}(x, y, r)+m_{2} f_{2}(x, y, r) & I_{2} f_{3}(x, y, r)+m_{2} f_{4}(x, y, r) \\
I_{2} f_{3}(x, y, r)+m_{2} f_{4}(x, y, r) & I_{1}+I_{2} f_{5}(x, y, r)+m_{2} f_{6}(x, y, r)
\end{array}\right]
$$




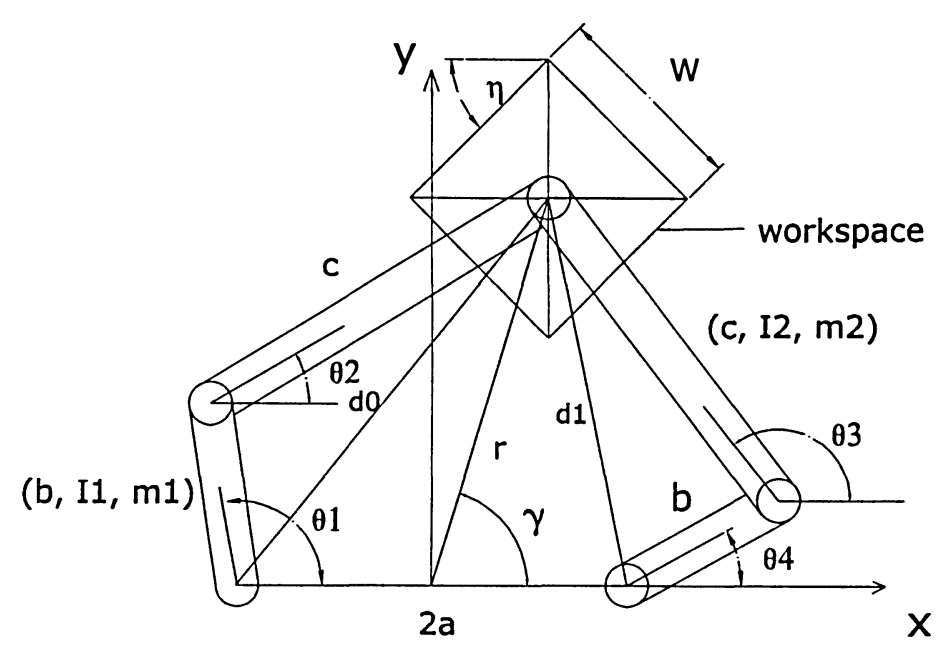

Figure 5.1: A 2DOF planar five-bar linkage

where

$$
\begin{aligned}
f_{1}(x, y, r) & =\left[P_{13}^{2}+P_{12}^{2}\right] \\
f_{2}(x, y, r) & =\left[b^{2}+2 X b \cos \left(\theta_{1}-\theta_{2}\right) P_{13}\right] \\
f_{3}(x, y, r) & =\left[P_{13} P_{43}+P_{12} P_{42}\right] \\
f_{4}(x, y, r) & =\left[P_{43} \cos \left(\theta_{1}-\theta_{2}\right)+P_{12} \cos \left(\theta_{4}-\theta_{3}\right)\right] X b \\
f_{5}(x, y, r) & =\left[P_{43}^{2}+P_{42}^{2}\right] \\
f_{6}(x, y, r) & =\left[b^{2}+2 X b \cos \left(\theta_{4}-\theta_{3}\right) P_{42}\right]
\end{aligned}
$$

Here, $\left(I_{1}, I_{2}, m_{2}\right)$ are inertial and $X, b$ are geometrical parameters appearing in inertia matrix of a planar five bar mechanism. It is notable to recognize that elbow link length denoted as $c$, mass of shoulder link denoted as $m_{1}$, and location of center of mass of elbow denoted as $X$ have no effect on the inertia matrix.

As it is shown in (5.26) also inertia matrix can be imagined as summation of two inertia matrices $M_{e}, M_{s}$ which represent the inertia matrices of elbows and shoulders relative to 
active joints respectively.

$$
\begin{gathered}
M=M_{s}+M_{e} \\
M_{s}=\left[\begin{array}{cc}
I_{1} & 0 \\
0 & I_{1}
\end{array}\right] \\
M_{e}=\left[\begin{array}{ll}
M e_{11} & M e_{12} \\
M e_{21} & M e_{22}
\end{array}\right] \\
\cdot \\
M e_{11}=I_{2} f_{1}(x, y, r)+m_{2} f_{2}(x, y, r) \\
M e_{12}=I_{2} f_{3}(x, y, r)+m_{2} f_{4}(x, y, r) \\
M e_{21}=M_{12} \\
M e_{22}=I_{2} f_{5}(x, y, r)+m_{2} f_{6}(x, y, r)
\end{gathered}
$$

where $f_{1}, f_{2}, f_{3}, f_{4}, f_{5}, f_{6}$ are defined by (5.20) to (5.26).

As it can be examined from the concept of isotropy, $M_{s}$ is diagonal and isotropic matrix. The non-isotropic behavior of the inertia matrix originates from the elbow links which are located in distance from the mechanism base and they cause Coriolis and Centrifugal disturbing acceleration which should be optimized for better isotropic behavior of the mechanism.

\subsection{Global Multi-Objective Minimax Optimization of a Planar Five-bar Haptic Device}

In this section, the culling algorithm and dynamic and kinematic dexterity measures are used to re-examine the five-bar linkage based haptic interface for both dynamic and kinematic conditioning. In Fig. (4.1), the general feature of five-bar linkage with a square workspace is shown. For an inertial optimization, it is assumed that the device is held with a light finger grip so hand inertia is neglected. Stocco optimized the mechanism due to kinematic 
conditioning and established the following symmetry and positioning guidelines for the device [3].

1. Center of square workspace is located on y axis. $\left(\gamma=90^{\circ}\right)$

2. The square workspace is parallel to $\mathrm{x}$ axis. $(\eta=0)$

3. The manipulator is symmetric to y axis. $\left(b_{0}=b_{1} ; c_{0}=c_{1}\right)$

4. The manipulator should not pass the singular points as it travels in the symmetric workspace. $\left(0 \leq \lambda_{0} \leq 180^{\circ} ; \quad 0 \leq \lambda_{1} \leq 180^{\circ}\right)[3]$.

Since the computation cost for global minimax algorithm is highly dependant on number of independent parameters, to avoid numerical complexities in matter of multi-objective optimization problems, it is necessary to have more simplified dynamic inertia in terms of number of independent variables [6]. In section 4.2 of this thesis, more symmetry and inertia guidelines were introduced to get to a more simplified model such as simplified inertia matrix derived in (5.3).

In this section, a new global multi-objective algorithm is introduced to meet the complexity problem of kinematic and dynamic conditioning of a planar five-bar mechanism. To get to best overall behavior from a reasonably small device, one should consider both kinematic and dynamic criteria simultaneously [6]. One option is to define a weighted function based on linear combination of kinematic and dynamic dexterity. Since the number of optimization parameter increases, such methods could be computationally expensive and because of different nature of kinematic and dynamic dexterity, sometime the interpretation of result will be really complex or even meaningless. To avoid the difficulties involved in creating a weighted performance function, the parameter space of kinematic optimization problem has to be optimized and its domain must be narrowed down to kinematic optimal space. Then this kinematical optimum parameter space will be assumed as a sub-optimal domain to dynamic conditioning optimization problem to get to a final multi-objective optimal re- 
sults. In other word, the output of kinematic analysis will be used as input for dynamic analysis and the method could be computationally expensive. As it was discussed in section 3.4, kinematically optimized 5-bar linkage has the "Elbow out" posture (when $r \geq 10 \mathrm{~cm}$ ) and the performance increases as $r \longrightarrow \infty$. The optimal link lengths are calculated by the culling algorithm and are illustrated as functions of $r$ in Fig. (4.2).

As shown in eqs. (5.11) to (5.14), inertia matrix of the whole the manipulator is function of four independent dynamic parameters $r, I_{1}, I_{2}, m_{2}\left(X_{2} X_{3} X\right.$ are assumed to be constant). The objective function for our optimization problem denoted as $S$ is the dynamic dexterity measure defined as:

$$
S\left(r, I_{1}, I_{2}, m_{2}\right)=\mathrm{DDM}=\frac{\sigma\left(\mathbf{J}^{-1} \mathbf{M}\right)}{\tilde{\sigma}\left(\mathbf{J}^{-1} \mathbf{M}\right)},
$$

where matrix $\mathbf{J}$ is Jacobian matrix, $\mathbf{M}$ is inertia matrix of the manipulator and $\underline{\sigma}$ and $\tilde{\sigma}$ represents minimum and maximum eigenvalues, respectively. As shown in eq. Finding the best worst case scenario of the objective function $\left(S^{*}\right)$ over entire workspace is our optimization problem.

$$
S^{*}=\max \min S\left(r, I_{1}, I_{2}, m_{2}\right)
$$

The culling algorithm is proposed as an effective optimization method for finding the best worst case scenario problem, specially in manipulator design [6]. Solving the optimization problem respective to 4 independent variable $\left(r, I_{1}, I_{2}, m_{2}\right)$ results in a trivial answer which is presented in following conditions.

$$
\begin{aligned}
& \text { - } r_{o p t} \longrightarrow \infty \\
& \text { - } m_{2 o p t} \longrightarrow 0 \\
& \text { - } I_{2 o p t} \longrightarrow 0
\end{aligned}
$$


- $I_{1 o p t} \longrightarrow \infty$

It is clear that the results are not feasible and physically can not be acceptable. To avoid such trivial answers, a new constrained multi-objective minimax optimization method is introduced in following section.

\subsection{Constraint Multi-objective Minimax Optimization of a Desktop Planar Five-bar Haptic Device}

As described in the previous section, conditions explained as trivial answers are the global optimum solution for multi-objective optimization of a planar 5-bar haptic device. These answers are obviously not feasible answers. It can easily be imagined that since $I_{2 o p t} \longrightarrow 0$ and $m_{2 o p t} \longrightarrow 0$, the optimum feasible answer must be the lowerbound of these two parameters and , similarly, since $r_{\text {opt }} \longrightarrow \infty$ and $I_{1 o p t} \longrightarrow \infty$, the upperbound of these parameters must be the optimum feasible answers for the multi-objective optimization problem. So simply these feasible answers can be written in the following equations.

$$
\begin{aligned}
r_{\text {opt }} & =r_{u p} \\
I_{1 o p t} & =I_{1-u p} \\
m_{2 o p t} & =m_{2-l o w} \\
I_{2 o p t} & =I_{2-l o w}
\end{aligned}
$$

In the next section, a design guideline is proposed to find the lower and upper bounds of the inertial parameters. Moreover, the analysis will be based on the main characteristics of a good quality desktop planar haptic device that was investigated by Hayward [7]. In [7], the rough guideline for its size, in term of its footprint, was proposed to be around the size of a book. For practical purposes, the inertia of the whole system should be smallest within different design choices considering the lowest possible weight for actuators. 


\subsection{Design Constraints For a Desktop 5-bar Haptic De- vice}

Since the whole mechanism should be constructed as a desktop device around size of a book, $r_{\text {opt }}$ is predictable and can be assume as $r_{\text {opt }}=25 \mathrm{~cm}$. The joint and rotor inertia is dominated by the inertia of the linkages which are made from circular cross-section 2024-T4 aluminum tubing of thickness $t$. Center of mass of the tube is in links geometric center and mass per unit length is a function of wall thickness $t$ and diameter $d$. Parameter $t$ is assumed to be constant and the minimum mass per unit length is calculated based on following equation to avoid resonance of the structure [3].

$$
\begin{aligned}
m_{\min } & =8.7 t d(\mathrm{gr} / \mathrm{cm}) \\
d & =5.1 \times 10^{-6}\left(b_{o p t}+c_{o p t}\right)^{2} \omega(\mathrm{cm})
\end{aligned}
$$

By having $m_{\min }$ as minimum mass per unit length, $m_{2-l o w}$ can be computed as:

$$
m_{2-l o w}=m_{\min } c_{o p t}
$$

Assuming the whole weight of the haptic system equals to $m_{\text {total }}, m_{1-u p}$ and consequently $I_{1-u p}$ can be easily calculated as follows:

$$
\begin{aligned}
m_{1}+m_{2} & =m_{\text {total }} \\
m_{1-u p} & =m_{\text {total }}-m_{2-\text { low }} \\
I_{1-u p} & =m_{1-u p} b_{o p t}^{2}
\end{aligned}
$$

By finding upperbound and lowerbound of respective parameter and by using eqs. (5.45) to (5.47) the problem of constrained multi-objective optimization of the five bar mechanism will be solved. It should be mentioned that the problem of finding optimum link length 
already solved in (4.2) and the solution is as follows :

$$
\begin{aligned}
& a_{o p t}=a\left(r_{o p t}\right) \\
& b_{o p t}=b\left(r_{o p t}\right) \\
& c_{o p t}=c\left(r_{o p t}\right)
\end{aligned}
$$

\subsection{Analysis of The Inertia Parameter On The Dy- namic Dexterity Measure (Dimensional Analysis)}

The dynamic equations for a planar five-bar haptic device is introduced through eqs (5.1) to (5.5). As it was already discussed, inertial behavior of such a mechanism is dependent on four variables $\left(r, I_{1}, I_{2}, m_{2}\right)$ assuming that the manipulator already been geometrically optimized to get to sub-optimal link length domain defined in Fig. (4.2). Therefore, the link length are functions of $\mathrm{r}$ and can be written as $a(r), b(r), c(r)$.

Since in evaluation of dynamic dexterity measure introduced in (2.34) only the ratio between eigenvalues are important, it can be proved that the actual dexterity measure is only dependant just on three variables $\left(r, \frac{m_{2}}{I_{1}}, \frac{I_{2}}{I_{1}}\right)$.

Validity of previous theorem can be easily shown by examining the different property of eigenvalues and eigenvectors of a square matrix in basic matrix calculus. The theorem is, if a matrix is multiplied by a diagonal matrix or a scalar value, all the eigenvalues will be multiplied by the same scalar but the ratio of the eigenvalues remains the same. As it can be seen in (5.1) to (5.5), the value $I_{1}$ can be factored as an scalar from the mass matrix as follows:

$$
M=I_{1}\left[\begin{array}{cc}
1+f_{1}(x, y, r) I 2+f_{2}(x, y, r) m 2 & f_{3}(x, y, r) I 2+f_{4}(x, y, r) m 2 \\
f_{3}(x, y, r) I 2+f_{4}(x, y, r) m 2 & 1+f_{5}(x, y, r) I 2+f_{6}(x, y, r) m 2
\end{array}\right]
$$




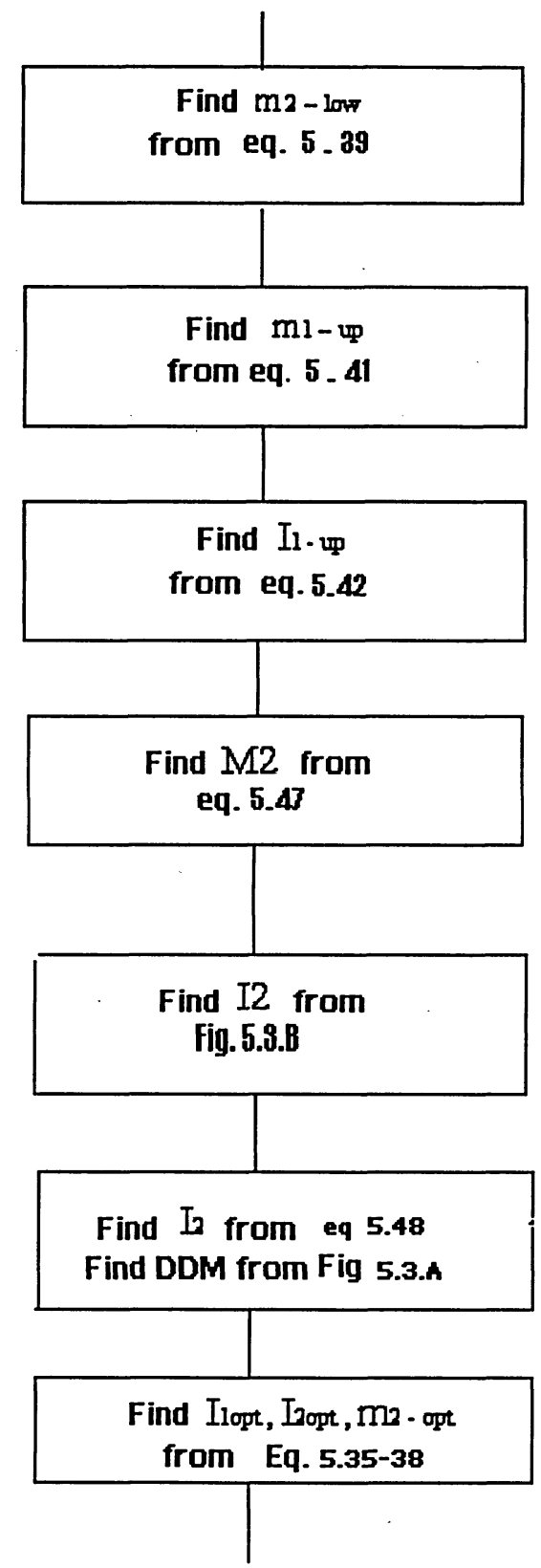

Figure 5.2: The proposed dynamic design flowchart of planar five-bar linkage 
where $I 2$ and $M 2$ are defined as following ratios:

$$
\begin{aligned}
M 2 & =\frac{m_{2}}{I_{1}} r^{2} \\
I 2 & =\frac{I_{2}}{I_{1}}
\end{aligned}
$$

It is proved from basic calculus that $I_{1}$, as a scalar, has no effect on the dynamic dexterity performance. Therefore, two dynamic ratios $M 2, I 2$ as dynamic parameters and $r$ as workspace location are main three dominant dynamic parameters. In the new proposed multi-objective optimization method, the output of kinematic optimization will be used. It means that link lengths are assumed to be function of $r$ Fig (4.2). Fig. (5.3B) shows the relation between $M 2$ and $I 2$ in different workspace locations. ( parameters $M 2$ and $I 2$ are dimensionless and the resulting figure will make more sense ). Also in Fig (5.3A) the relation between the $D D M$ as dynamic dexterity performance relative to $M 2$ is illustrated for different desktop sizes and results for $r=20 \mathrm{~cm}$ is presented in Table 5.1. The width of workspace is fixed to $10 \mathrm{~cm}$ and the resolution is $\Delta(w)=0.1$. Also the dexterity and the manipulability for a non-optimal and optimal dynamic parameter was shown in Fig. (5.4) and Fig. (5.5) during the process of the optimization. $I_{1}=90$ gr.cm ${ }^{2} ; I_{2}=1$ gr.cm ${ }^{2} ; m_{2}=.8 \mathrm{gr} ; \mathrm{r}=25 \mathrm{~cm}$ are the dynamic parameters in Fig. (5.4) and $I_{1}=100$ gr.cm ${ }^{2} ; I_{2}=1$ gr.cm ${ }^{2} ; m_{2}=.8 \mathrm{gr} ; r=25 \mathrm{~cm}$ are the dynamic parameters in Fig. (5.5). Also flowchart for the proposed design process, introduced in section 5.4, is presented in Fig. (5.2).

\subsection{Case Study with New Method}

In this section, we are trying to design an actual planar five-bar haptic device through the new design method explained in the section 5.4. The total mass for the desktop device is assumed to be $1000 \mathrm{gr}$ constructed from circular cross section 2024-T4 aluminum tubing with 


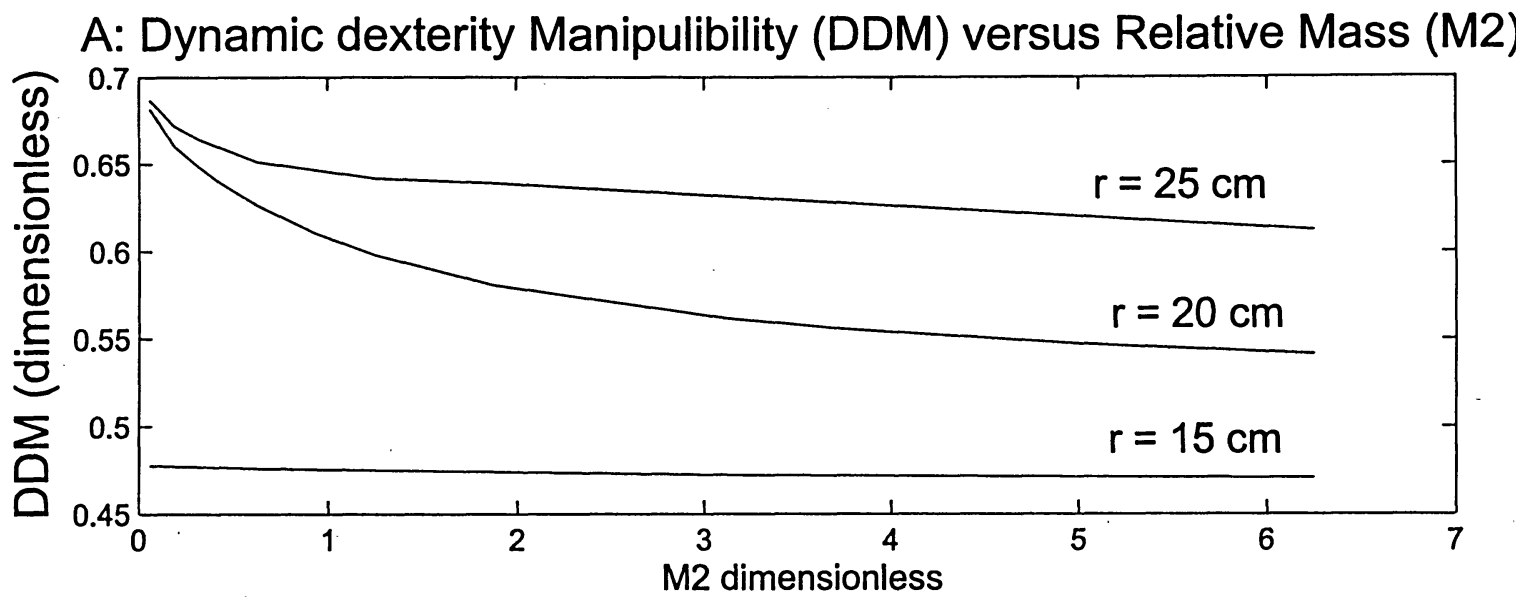

B: Relative Inertia (12) versus Relative Mass (M2)

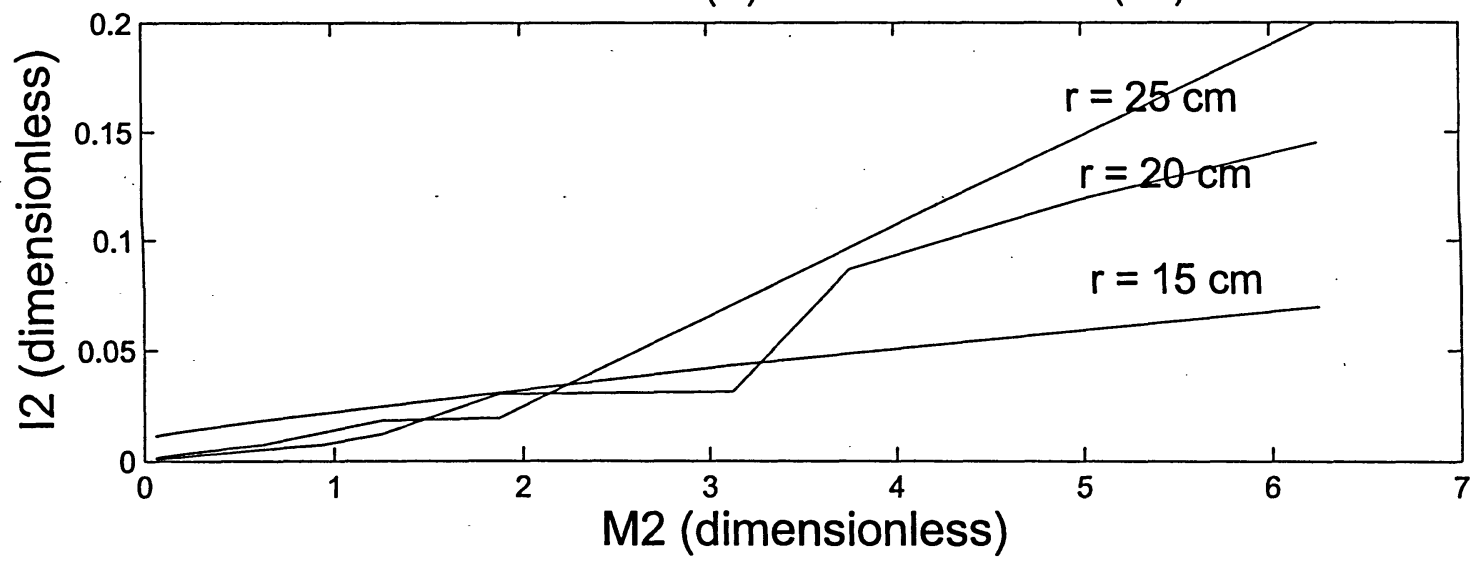

Figure 5.3: Proposed dimensionless curves for kinematic and dynamic optimization of five-bar mechanism. 
Table 5.1: Result of proposed dimensionless optimization analysis of five-bar linkage for $r=20 \mathrm{~cm}$.

\begin{tabular}{|c|c|c|}
\hline$M 2=\frac{m_{2}}{I_{1}} r^{2}$ dimensionless & $I 2=\frac{I_{2}}{I_{1}}$ dimensionless & DD dimensionless \\
\hline 6.25 & .145 & .5412 \\
\hline 5 & .119 & .5469 \\
\hline 3.75 & .087 & .5553 \\
\hline 3.125 & .031 & .5616 \\
\hline 1.875 & .03 & .5809 \\
\hline 1.25 & .012 & .5980 \\
\hline .9375 & .007 & .6103 \\
\hline .625 & .005 & .6265 \\
\hline .4375 & .0035 & .6390 \\
\hline .3125 & .0026 & .6488 \\
\hline .1875 & .0016 & .6603 \\
\hline .0625 & $1.05 \mathrm{e}-5$ & .6815 \\
\hline
\end{tabular}
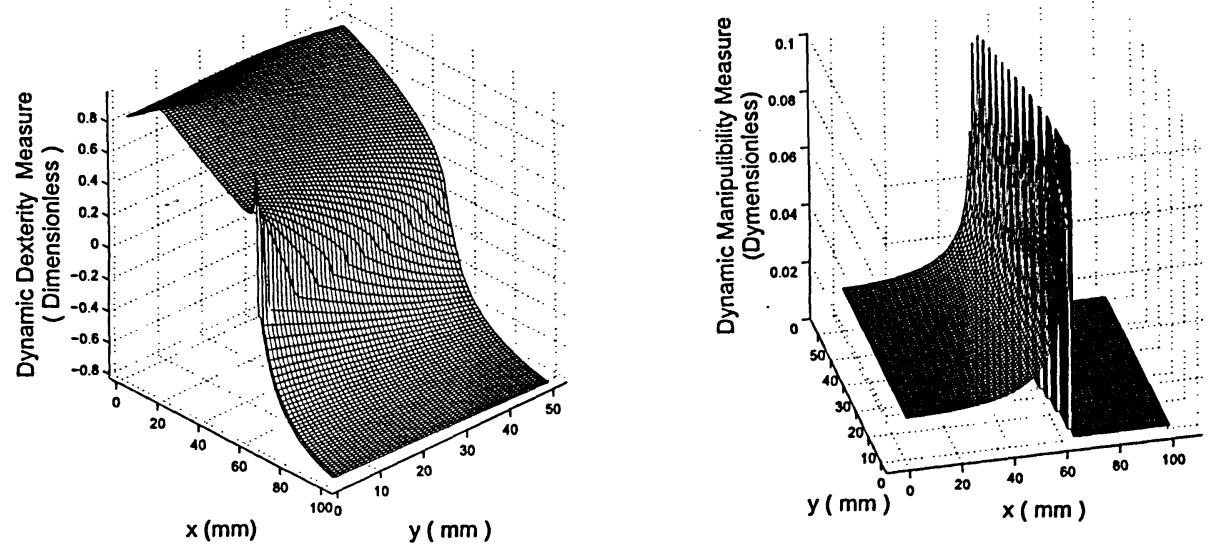

Figure 5.4: Dexterity for a non-optimal dynamic parameter; 

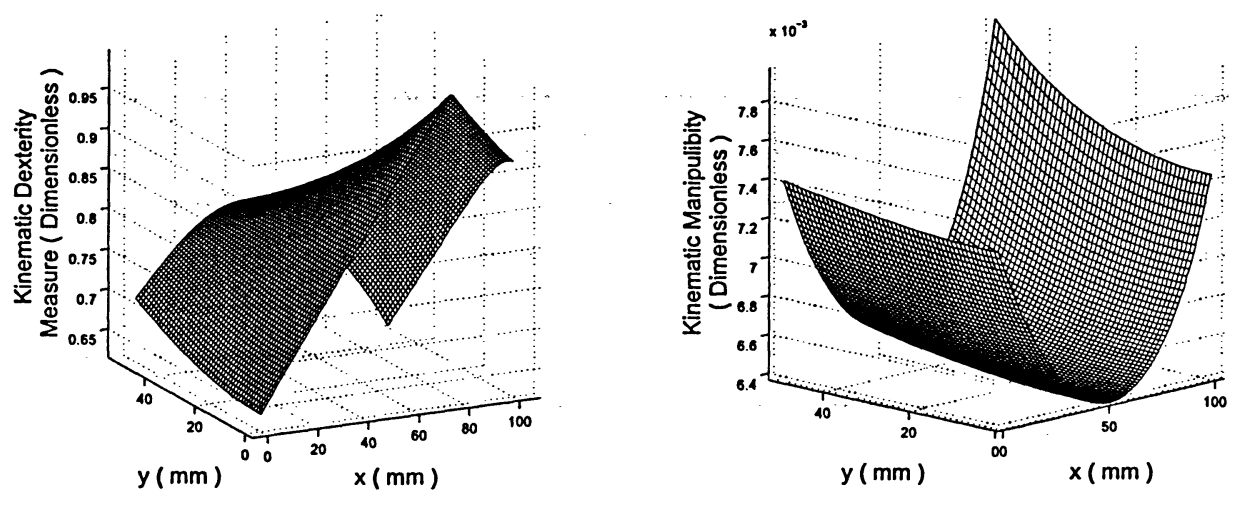

Figure 5.5: Dexterity for an optimal dynamic parameter;

wall thickness $t=2 \mathrm{~mm}$. In Stocco's research the first natural frequency of the mechanism is assumed to be $\omega=200 \pi$ as a design parameter and the desktop size is around $30 \mathrm{~cm} \mathrm{[3].}$ In order to be able to compare the results in this thesis and Stocco method, the same design parameter will be assumed throughout this research. $r_{\max }$ is $25 \mathrm{~cm}$ and the elbow length is found to be $c=25 \mathrm{~cm}$ from the Fig. (4.2). Minimum mass per unit length is computed from eqs. (5.39) and (5.40) by considering $c_{\min }=25 \mathrm{~cm}$ and $b_{\min }=13 \mathrm{~cm}$ from the Fig. (4.2).

$$
\begin{aligned}
& d=(5.1) 10^{-6}(13 \mathrm{~cm}+25 \mathrm{~cm})^{2}(200 \pi)=.93 \quad \mathrm{~cm} \\
& m_{\min }=8.7 \frac{\mathrm{gr}}{\mathrm{cm}^{3}}(.2 \mathrm{~cm}) 4.62=1.6 \mathrm{gr} / \mathrm{cm}
\end{aligned}
$$

Then $m_{2-l o w}$ can be calculated as:

$$
\begin{aligned}
& m_{2-l o w}=m_{\min } c=1.6(25)=40 \mathrm{gr} \\
& m_{1-u p} \text { is computed from }(5.43) . \\
& m_{1-\text { up }}=m_{\text {total }}-m_{2-\text { low }}=1000-40=960 \mathrm{gr}
\end{aligned}
$$

from (5.44) we can derive the $I_{1-u p}$ :

$$
I_{1-u p}=m_{1-u p} b^{2}=960 *(13)^{2}=162,240 \quad \mathrm{gr} \mathrm{cm}^{2}
$$


It is concluded from (5.37) that $m_{2 o p t}=m_{2-l o w}=40 \mathrm{gr}$ and $I_{1 o p t}=I_{1-u p}=162,240 \mathrm{gr}$.

By evaluating the parameter $M 2$ by eq. (5.49) we can calculate $I 2$ and consequently $I_{2 o p t}$ from eq. (5.50).

$$
\begin{aligned}
& M 2=m 2_{\text {opt }} r^{2}=\frac{m_{2 o p t}}{I_{1 o p t}} r^{2}=\frac{40}{162,240}(25)^{2}=.1541 \text { dimensionless } \\
& I 2=.87 \\
& I_{2 o p t}=I 2 I_{1 o p t}=(.87)(162,240)=141,000 \mathrm{gr} \mathrm{cm}^{2}
\end{aligned}
$$

Therefor at the end the optimum parameters are introduced as follows:

$$
\begin{aligned}
& r_{\text {opt }}=25 \mathrm{~cm} \\
& I_{1 \text { opt }}=162,240 \mathrm{gr} \mathrm{cm}^{2} \\
& I_{2 o p t}=141,000 \mathrm{gr} \mathrm{cm}{ }^{2} \\
& m_{2 o p t}=40 \mathrm{gr} \text { and } m_{1}=960 \mathrm{gr}
\end{aligned}
$$

Dynamic dexterity performance $=.68$ (dimensionless)

\subsection{Case Study with Stocco's Multi-objective Optimiza- tion Method}

Stocco [3] proposed a multi-objective one-dimensional optimization method based on dynamic and kinematic conditioning. The method picks up the kinematically optimized geometric parameters for different values of $r$ Fig (4.2) and then tries to find configurations with best dynamic dexterity performance. Masses of links are calculated from (5.39) and (5.40) and assuming a uniform cross section of aluminum tubing for link structure, the mass moment of inertia of link relative to its end point can be calculated from equation $I=\frac{1}{3} m l^{2}$. All geometric parameters $(a, b$ and $c$ ) are chosen as a function of $\mathrm{r}$ from Fig.(4.2) and just $\mathrm{r}$ is the only independent variable. After evaluation of dynamic dexterity introduced in (2.34), the maximum value for its performance can be easily found from Fig. (5.6) and the optimum geometric parameters are: 


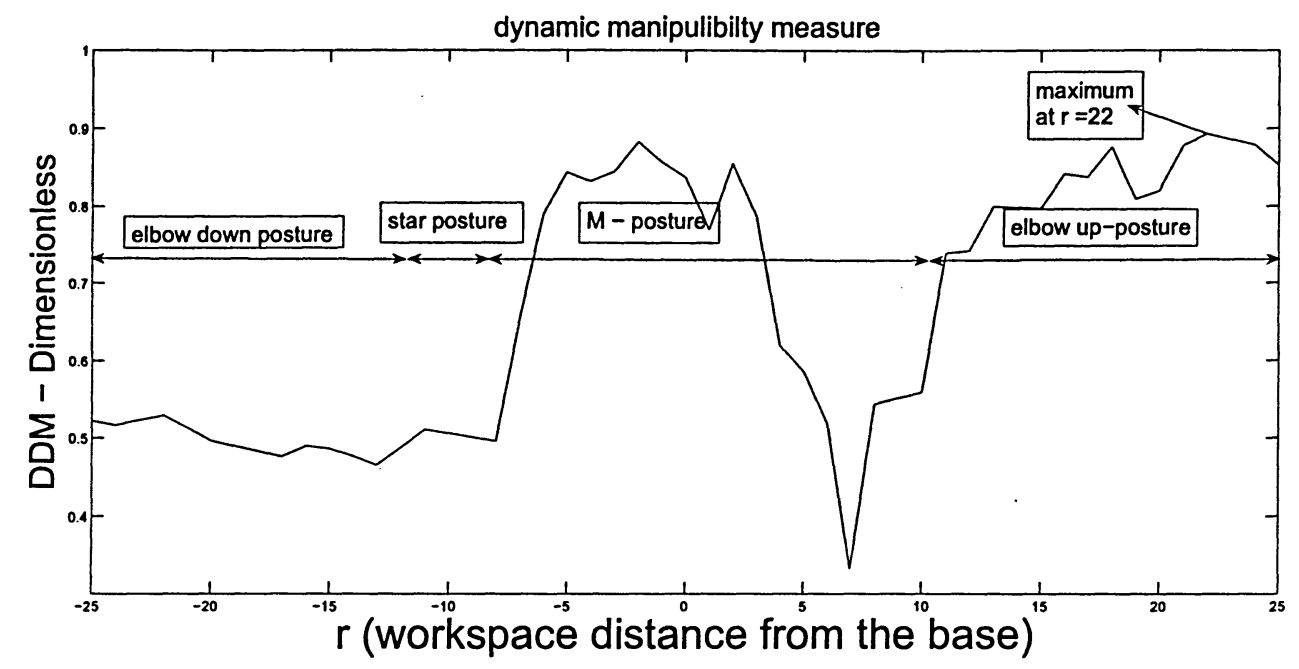

Figure 5.6: Multi-objective optimization results from stocco method.

$r_{\text {opt }}=22 \mathrm{~cm} ; a=7.5 \mathrm{~cm} ; b=11.5 \mathrm{~cm} ; c=21 \mathrm{~cm}$,

By evaluating mass per unit length from (5.39) and (5.40) the other dynamic parameter can easily be computed as follows:

$$
\begin{aligned}
& \mathrm{d}=2.55 e-6(11.5 \mathrm{~cm}+21 \mathrm{~cm}+11.5 \mathrm{~cm}+21 \mathrm{~cm}) 200 \pi=6.77 \mathrm{~cm} \\
& \mathrm{~m}=8.7(.2)(6.77)=11.78 \mathrm{gr} / \mathrm{cm} \\
& \mathrm{m}_{2}=c \mathrm{~m}=21(11.78)=247.4 \mathrm{gr} \\
& \mathrm{m}_{1}=b \mathrm{~m}=(11.5)(11.78)=135.47 \mathrm{gr} \\
& I_{2}=\frac{1}{3} \mathrm{~m}_{2} c^{2}=\frac{1}{3}(247.4)\left(21^{2}\right)=11,329 \mathrm{gr} \mathrm{cm}^{2} \\
& I_{1}=\frac{1}{3} \mathrm{~m}_{1} b^{2}=\frac{1}{3}(135.47)\left(11.5^{2}\right)=11,329 \mathrm{gr} \mathrm{cm}^{2}
\end{aligned}
$$

Dynamic dexterity performance $=.91$ 
Table 5.2: Comparison of Optimized 5 bar linkage with Stocco and the new method. Unit of length, mass and inertia are $\mathrm{cm}, \mathrm{gr}$ and $\mathrm{gr} \mathrm{cm}^{2}$ respectively. Dexterity measures are unitless.

\begin{tabular}{|c|c|c|c|c|c|c|c|c|c|}
\hline Optimization methods & $r_{\text {opt }}$ & $a_{\text {opt }}$ & $b_{\text {opt }}$ & $c_{\text {opt }}$ & $I_{\text {lopt }}$ & $I_{2 o p t}$ & $m_{2 o p t}$ & $K D M$ & $D D M$ \\
\hline The proposed method & 25 & 7.5 & 11.5 & 21 & 162 & 141000 & 40 & .73 & .68 \\
\hline Stocco's method & 22 & 9 & 13 & 25 & 53561 & 36309 & 247 & .67 & .52 \\
\hline
\end{tabular}

\subsection{Comparison Of Proposed Method With Stocco's Method}

As it is shown in table 5.2 by applying the new multi-objective method proposed on the manipulator with the same dynamic and kinematic parameters, the actual dynamic performance will be calculated as $.5243 \mathrm{~cm}$ which is too far from .6810 which is the dexterity performance calculated by our new multi-objective method. Kinematic dexterity measure obtained from the new method is also better than Stocco method. Therefore, it can be concluded that the optimized 5 bar linkage with proposed method is more dexterous both kinematically and dynamically comparing to the manipulator optimized by Stocco Method.

Since Stocco method is dynamically a one dimensional multi-objective optimization technique along parameter $\mathrm{r}$, parameter space is very small and the method ignores more possible dynamic optimization along other main dynamic parameters such as $\left(I_{1}, I_{2}, m_{2}\right)$ resulting poor outputs. But the optimization technique presented in this thesis is a four dimensional multi-objective optimization technique along four parameter $\left(r, I_{1}, I_{2}, m_{2}\right)$. Parameter space is big enough and the results are more liable.

Moreover, Stocco proposed Fig. (4.2) for kinematic design of 5 bar linkage and there is a high need for the respective optimization curves in dynamic studies. Presenting Fig. (5.3) and Table 5.1 as dynamic optimization results, in dimensionless form, could be one of the major contributions of this research which was totally ignored by Stocco's Research. 
Dynamic Manipulibility Measure

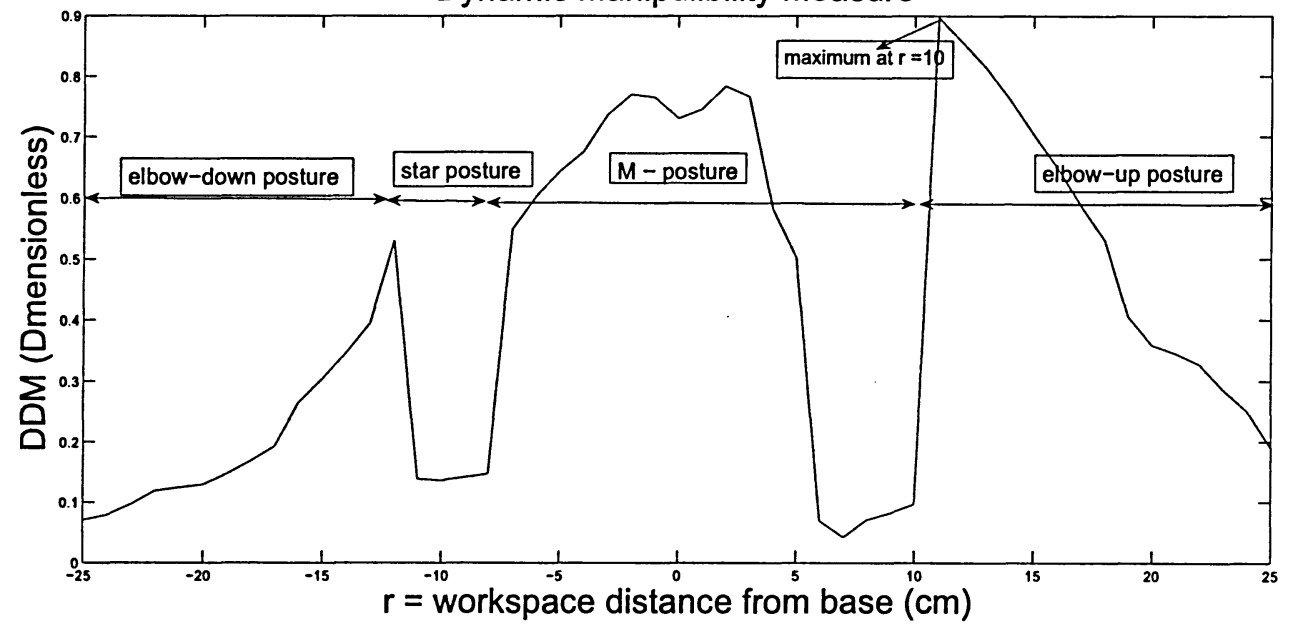

Figure 5.7: Multi-objective optimization results from Stocco dynamic dexterity definition 


\section{Chapter 6}

\section{CONCLUSIONS}

\subsection{Contributions}

This chapter outlines the main contributions in this thesis and also some the challenging areas for future works is introduced. The contributions are summarized as follows:

- The exact inertia matrix for 5-bar planar haptic device.

Comparing to other works [3] [11], this research uses the exact inertia matrix for the five-bar planar manipulator [34] and the optimization results are more realistic due to having a good dynamical model for the system.

- Dimensional analysis of dynamic and geometric parameters.

By using dimensional analysis, number of inertial parameters $\left(I_{1}, I_{2}, m_{2}\right)$ reduced from three dependent variables to just two dimensionless independent variables $(I 2, M 2)$ introduced in section 5.5 .

- The global multi-dimensional multi-objective optimization technique.

As in other technique introduced by Stocco [3], there was a coupling between kinematic and dynamic parameters, due to (5.37) and (5.38), and these method can be 
imagined more constrained optimization method rather than a global techniques. In the new techniques proposed the relation between geometrical parameter and dynamic parameters is removed and more number of dynamical parameter is explored to get to actual optimum parameters. Hence, the result of these optimizations are more close to a global optimum result than stocco multi-objective method.

Moreover, due to the fact that, the new global multi-objective optimization techniques has a non-feasible trivial result, a new constrained techniques is developed by application of design guidelines to avoid unrealistic results. In other word, since the global optimum is not achievable in reality, the closest parameters is selected as the feasible optimum results.

- New design procedure and guidelines for five-bar linkage haptic device. A new design procedure for designing an dynamical and kinematical optimized desktop size haptic device is presented in section (5.4) and Fig. (5.2). The main objective is to protect the device from resonance [7] [3] and consequently structural failure. Also in order to have a more fast-dynamic response system, the inertia of the system has to be minimized [7].

\subsection{Future work}

The future proposed works for dynamic and kinematic research of a five-bar linkage can be summarized as follows:

- Effect of natural frequency of five-bar mechanism on the design.

A very rough estimation of minimum natural frequency response is used by stocco [7] [3] as an additional design parameter. The mass per unit length is assumed to be uniform which is not an assumption in the new design method introduced in this thesis. 
Therefore, more attempt is needed to have a better estimate of vibrational behavior of a five-bar haptic device with a non-uniform mass per unit length. Then we could use the results as more realistic constraint for finding a more realistic manipulator for industrial applications.

- Application of the new technique on more complex manipulators with different dexterity measures.

From point of view of engineering design for constructing a more dynamically fastresponse device, minimizing total mass of the whole mechanism is very important [7] [3]. Stocco introduced new dynamic dexterity measure to meet this requirement and proposed a new performance measure as $\frac{1}{1+\tilde{\sigma}}$ which $\tilde{\sigma}$ is the biggest eigenvalue of the dynamic dexterity matrix introduced in (2.34). The multi-objective optimization results is illustrated in Fig. (5.6) which is completely different from results in Fig. (5.5). Implementation of the Stocco dynamic dexterity with the new proposed algorithm in this thesis can be another interesting and challenging problem . Also application of the new method on more complex haptic devices such as twin-pantograph haptic pen or twin-pantograph hybrid manipulator [3] [2] [22] could be a good challenge for our new optimization strategy.

- More theoretical and computational base sensitivity studies of all new optimization strategies in different kinematical and dynamical parameters and domains.

Since in our optimization strategy all parameters discredited and some of them assumed to be constant and there is lot of coupling between these variables and moreover just limited domain of parameters is selected during optimization process, so basically there is no guarantee for generalizing the results to other parameter domain,unless by establishing a convergence analysis independent than discritization and parameter 
dimensions and domains [6]. 


\section{References}

[1] J.P. Karidis, G. MeVicker, J.P. Pawaletko, M. Goldowsky, R.E. Brown and R.R. Comulada, "The Hummingbird Minipositioner - Providing Three-Axis Motion at 50 G's With Low Reactions" Proc. IEEE International Conference on Robotics and Automation (Nice, France), Vol. 1, pp. 685-692, May 10-15., 1992.

[2] S. E. Salcudean and L. Stocco, "Isotropy and Actuator Optimization in Haptic Interface Design", Proc. IEEE International Conference on Robotics and Automation ( San Francisco, CA), Vol. 1, pp. 763-769, April 22-28, 2000.

[3] L. Stocco, S. E. Salcudean and F. Sassani, "Fast Constrained Global Minimax Optimization of Robot Parameters", Robotica, Vol. 16, pp. 595-605, Nov, 1998.

[4] P. Chiacchio, S. Chiaverini, L. Sciavicco, and B. Siciliano, "Reformulation of Dynamic Manipulabilty Ellipsoid for Robotic Manipulators", Proc. IEEE International Conference on Robotics and Automation, (Sacramento, CA, USA), Vol. 3, pp. 2192-2197, 9-11 April, 1991.

[5] O. Ma and J. Angeles, "Optimum Design of Manipulators Under Dynamic Isotropy Conditions", Proc. IEEE International Conference of Robotics and Automation (Atlanta, Georgia), Vol. 1, pp. 470-475, 2-6 May , 1993.

[6] L. J. Stocco, S. E. Salcudean and F. Sassani, " Mechanism Design For Global Isotropy With Applications to Haptic Interfaces", The Winter Annual Meeting of the 
ASME. Sixth Annual Symposium on Haptic Interfaces for Virtual Enviroment and Teleoperator Systems. Dallas, Texas, pp. 115-122, Nov. 15-21, 1997.

[7] V. Hayward, J. Choksi, Gonzalo and C. Ramstein, "Design and Multi-Objective Objective Optimization of a Linkage for a Haptic Interface", Proc. ARK 94. 4th International Workshop on Advances in Robotic Kinematics (Ljubliana, Slovenia), pp. 352-359, June 1994.

[8] O. Ma and J. Angeles, "The Concept of Dynamic Isotropy and Its Applications to Inverse Kinematics and Trajectory Planning ", Proc. IEEE International Conference on Robotics and Automation, (Cincinnati, OH, USA), Vol. 1, pp. 481-486, May 13-18, 1990.

[9] M. Sirouspour, S. DiMaio, S. Salcudean, P. Abolmaesumi and C. Jones, "Haptic Interface Control-Design Issues and Experiments with a Planar Device ", Proc. IEEE International Conference on Robotics and Automation, (San Francisco, CA, USA) ,Vol. 1, pp. 789-794, 2000.

[10] D.N. Nenchev, M. Uchiama, "Singularity-Consistent Path Planning and Control of Parallel Robot Motion Through Instantaneous-Self-Motion Type Singularities ", International Conference on Robotics and Automation, (Minneapolis, Minnesota, USA), Vol. 2, pp. 1664-1870, 22-28 April, 1996.

[11] T. Yoshikawa, "Manipulability of Robot Mechanisms ", The International Journal of Robotics Research, Vol. 4, No. 2, pp. 3-9, 1985.

[12] S. L. Chiu, "Task Compatibility of Manipulator Postures", The International Journal of Robotics Research, Vol. 7, No. 5, pp. 13-21, Oct 1988. 
[13] T. Yoshikawa, "Dynamic Manipulability of Robot Manipulators ", Journal of Robotic Systems, Vol. 2, No. 1, pp. 113-124, 1985.

[14] P. Chiacchio, S. Chiaverini, L. Sciavicco and B. Siciliano, "Influence of Gravity on the Manipulibility Elipsooid for Robot Arms ", ASME Journal of Dynamic Systems, Measurement and Control, Vol. 114, No. 4, pp. 723-727, 1992.

[15] M. T. Rosenstein and R. A. Grupen, "Velocity-Dependent Dynamic Manipulability ", Proc. IEEE International Conference on Robotics and Automation, (Washington DC) , Vol. 3, pp. 2424-2429, May 2002.

[16] P. Chiacchio, "A New Dynamic Manipulibity Ellipsoid for Redundant Manipulators", Robotica, Cambridge University Press, Vol. 18, pp. 381-387, 2000.

[17] T. Yoshikawa, Ralf Koeppe, "Dynamic Manipulibility Analysis of Compliant Motion ", Proc. IEEE International Conference on Intelligent Robots and Systems, (Granoble, France), Vol. 3, pp. 1472-1478, July- sep. 1997.

[18] J.B. Hunt, "Vibrational Analysis of Dynamic Systems" Mechanical Engineering Publication, 1979.

[19] J. Karidis, G. McVicker, J. Pawletko, L. Zai, M. Goldowsky, R. Brown and R. Comulada, "The Hummingbird Miniposisioner-Providing Three-Axis Motion at 50 G's With Low Reactions" (Nice, France), Proc. IEEE International Conference of Robotics and Automation, pp. 685-692, May 10-15, 1992.

[20] M. W. Spong and M. Vindyasagar, "Robot Dynamics and Control", John Wiley and Sons, New York, 1989. 
[21] L. Sciavicco and B. Siciliano, "Modelling and Control of Robot Manipulators", Springer, (Glascow, Scottalnd, UK), Sep. 2000.

[22] L. J. Stocco, S. E. Salcudean, F. Sassani, "Optimal Kinematic Design of a Haptic Pen", IEEE/ASME Transactions on Mechatronics, Vol. 6, No. 3, pp. 210-220, Sep. 2001.

[23] L. Stocco, S. E. Salcudean and F. Sassani, "Matrix Normalization for Optimal Robot Design ", Proc. IEEE International Conference on Robotics and Automation, (Leuven, Belgium), May 16-21, 1998.

[24] L. J. Stocco, S. E. Salcudean, F. Sassani, "On the Use of Scaling Matrices for TaskSpecific Robot Design ", Proc. IEEE Transaction on Robotics and Automation,(Vancouver, BC) vol. 15, no. 5, pp. 958-965, Oct., 1999.

[25] G. M. Griner, "A Comparison of Simulated Evolution and Genetic Evolution Performance", Proc. IEEE Conferencce on Evolutionary Computation, IEEE World Computational Intelligence, (Orlando, Florida), June 27-29, 1994.

[26] J. W. Herrmann, "A Genetic Algorithm for Minimax Optimization Problems", Proc. 1999 Congress on Evolutionary Computation, (Washington, DC, USA) Vol. 2, pp. 1099-1103, July 1999.

[27] S. Khatami, F. Sassani, "Isotropic Design Optimization of Robotic Manipulators Using a Genetic Algorithm Method", Proc. IEEE, International Symposium of Inteligent Control, (Vanchouver, Canada), pp. 562-567, Oct 27-30, 2002.

[28] M. Srinivas and L.M. Patnaik, "Genetic Algorithm: A Survey ", Computer, Vol. 27, series 6, pp. 17-26, June 1994. 
[29] Chan Li Theingi, I-Min Chen, "Cases Study on the Singularity of Five-bar Planar Mechanism with Differential Gear Drives ", The 6th Proc. International Conference on Engineering Design and Automation, (Maui, Hawaii), 2002.

[30] G. Alici, "Determination of Singularity Contours for Five-bar Planar Parallel Manipulators", Robotica, Vol. 18, pp. 569-575, 2000.

[31] P. Voglewede and Ebert-Uphoff, "Two Viewpoints on the Unconstrained Motion of Parallel Manipulators at or near Singular Configurations", Proc. International Conference on Robotics and Automation, (Atlanta, GA), Vol. 1, pp. 503-510, May 2002.

[32] V. K. Chan, I. Ebert-Uphoff, "Investigation of the Deficiencies of Parallel Manipulators in Singular Configurations Through the Jacobian Nullspace", Proc. IEEE International Conference on Robotic and Automation,(Seol, Korea), Vol. 2, pp. 1313-1320, 2001.

[33] F. Janabi-Sharifi "Kinematic Analysis of Planar Five-bar Mechanism" Technical Report, Robotic and Automation Lab., Mechanical engineering Dep., Ryerson University, Toronto, Ontario, 2003

[34] H. Kazerooni J. Guo, "Direct-Drive and Active Compliant End-Effector" (Active RCC), Proc. International Conference on Robotics and Automation, pp. 758-766, 1987. 


\section{Appendix A \\ Permissions}

Following is the permission email for Fig. (4.2). 


\section{Corrections}

- P. 35, line 6: "...... and the planar ..." should be changed to "...and a planar...".

- P.35, line 6: "...manipulators ..." should be changed to "...manipulator...".

- P.35, line 10: "... Kazerooni ..." should be changed to "...Kazerooni and J. Guo...".

- P.36, line 13: “...the end point..." should be change to "...base of each link...".

- P.36, line 15: "...end point ..." should be changed to "...base...".

- P.36, line 20: “... $\theta_{1}, \theta_{4} \ldots$ " should be changed "... $\theta_{2}, \theta_{3} \ldots$.."

- P.36, line 20: “... Symmetric ..." should be changed to "... geometrically symmetric".

- P.41, line 18: “...variable ..." should be changed to "...variables...".

- P.41, line 18: “...answer ..." should be changed to "... answer set...”.

- P.42, line 1: "...acceptable..." should be changed to "... realized.".

- P.42, line 19: “...Hayward..." should be changed to "...Hayward et al.".

- P.43, line 2: "...assume..." should be changed to "...assumed...".

- P.43, line 2: " $\ldots r_{\text {opt }}=25 \mathrm{~cm}$." should be changed to "... $r_{\text {opt }}=r_{u p}=25 \mathrm{~cm}$.".

- P.44, line 15: “...theorem..." should be changed to "... fact...".

- P.44, line 16: “...The theorem is ..." should be changed to "... It is shown that...".

- P.44, line 21: “...m2..." should be changed to “... $\frac{M 2}{r^{2}}$...".

- P.45, Fig. 5.2: 5.38, 5.41, 5.42, 5.47 and 5.50 should be changed to 5.41, 5.43, 5.44, 5.49 and 5.50 respectively.

- P.52, line 1: "As it is shown in the table 5.2 by..." should be changed to "By using our optimization code and ..."

- P.52, line 7: Stocco should be changed to Stocco's.

- P.58, line 19: “... The Winter..." should be changed to “... Proc. the Winter...”. 